Comment. Math. Helv. 75 (2000) 504-533

(C) 2000 Birkhäuser Verlag, Basel

0010-2571/00/030504-30\$1.50+0.20/0

Commentarii Mathematici Helvetici

\title{
Gromov hyperbolicity and the Kobayashi metric on strictly pseudoconvex domains
}

\author{
Zoltán M. Balogh* and Mario Bonk**
}

\begin{abstract}
We give an estimate for the distance function related to the Kobayashi metric on a bounded strictly pseudoconvex domain with $C^{2}$-smooth boundary. Our formula relates the distance function on the domain with the Carnot-Carathéodory metric on the boundary. The estimate is precise up to a bounded additive term. As a corollary we conclude that the domain equipped with this distance function is hyperbolic in the sense of Gromov.
\end{abstract}

Mathematics Subject Classification (2000). Primary 32H15, Secondary 32F15.

Keywords. Pseudoconvex domains, Kobayashi metric, Gromov hyperbolicity.

\section{Introduction: Notation and statement of results}

The Kobayashi metric is an important invariant metric that has been used for the study of holomorphic maps and function spaces in several complex variables. In general there is no exact formula for this metric but its boundary behavior has been extensively studied by several authors. For the latest results for strictly pseudoconvex domains we refer to [M1], [M2], [M3], [Fu]. A general survey on biholomorphically invariant metrics can be found in [JP]. These results are quite complete, however they have a local character as they provide sharp estimates of the differential metric near the boundary. Much less is known about the boundary behavior of the distance function - the integrated version of the metric. Partial results in this sense are included in the works of $[\mathrm{Ab}],[\mathrm{Al}],[\mathrm{FR}]$. We refer to $[\mathrm{K}]$ for a recent account of the subject. The content of these results are estimates, given for the distance of two points in certain special situations depending on the relative position of the points. The lack of global estimates of the distance function for two arbitrary points lies in the difficulty of determining the "almost geodesics" connecting two points in general relative position. In the present paper we overcome this difficulty by the principles of the theory of Gromov hyperbolic

\footnotetext{
* Supported by the Swiss National Science Foundation.

** Supported by a Heisenberg fellowship of the Deutsche Forschungsgemeinschaft.
} 
spaces. Our results are of global nature: we give a formula that describes the large scale structure of a strictly pseudoconvex domain equipped with the distance function related to the Kobayashi metric. As a consequence we obtain that the domain is hyperbolic in the sense of Gromov.

Let us start by fixing the notation we shall use throughout this paper. Suppose $\Omega \subseteq \mathbf{C}^{n}, n \geq 2$, is a bounded, strictly pseudoconvex domain with $C^{2}$-smooth boundary $\partial \Omega$. For a point $x \in \mathbf{C}^{n}$ let $\delta(x)=\operatorname{dist}(x, \partial \Omega)$ be the Euclidean distance of the point to the boundary of $\Omega$, and consider the signed distance function $\rho: \mathbf{C}^{n} \rightarrow \mathbf{R}$,

$$
\rho(x)=\left\{\begin{array}{rll}
-\delta(x) & \text { for } \quad x \in \Omega, \\
\delta(x) & \text { for } \quad x \in \mathbf{C}^{n} \backslash \Omega .
\end{array}\right.
$$

Then $\rho$ is $C^{2}$-smooth in an open neighborhood $N_{\epsilon}(\partial \Omega):=\left\{x \in \mathbf{C}^{n}: \delta(x)<\epsilon\right\}$ of $\partial \Omega$, and we have $\Omega=\left\{x \in \mathbf{C}^{n}: \rho(x)<0\right\}$. The tangent space $T_{p} \partial \Omega$ for $p \in \partial \Omega$ is given by $T_{p} \partial \Omega=\left\{Z \in \mathbf{C}^{n}: \operatorname{Re}\langle\bar{\partial} \rho(p), Z\rangle=0\right\}$, and its maximal complex or "horizontal" subspace is $H_{p} \partial \Omega=\left\{Z \in \mathbf{C}^{n}:\langle\bar{\partial} \rho(p), Z\rangle=0\right\}$, where $\bar{\partial} \rho(p)=\left(\frac{\partial \rho}{\partial \bar{z}_{1}}(p), \ldots, \frac{\partial \rho}{\partial \bar{z}_{n}}(p)\right)$, and $\langle Z, W\rangle=\sum_{\nu=1}^{n} Z_{\nu} \bar{W}_{\nu}$ is the standard Hermitian product of two vectors $Z=\left(Z_{1}, \ldots, Z_{n}\right)$ and $W=\left(W_{1}, \ldots, W_{n}\right)$ in $\mathbf{C}^{n}$.

By definition, the strict pseudoconvexity of $\Omega$ means that the Levi form $L_{\rho}(p ; \cdot)$ defined by

$$
L_{\rho}(p ; Z)=\sum_{\nu, \mu=1}^{n} \frac{\partial^{2} \rho}{\partial z_{\nu} \partial \bar{z}_{\mu}}(p) Z_{\nu} \bar{Z}_{\mu} \quad \text { for } \quad Z=\left(Z_{1}, \ldots, Z_{n}\right) \in \mathbf{C}^{n}
$$

is positive definite on $H_{p} \partial \Omega$ for $p \in \partial \Omega$. In particular, this implies the contact property of the horizontal bundle $H \partial \Omega$. Consequently one can define a metric $d_{H}$ - the horizontal, or Carnot-Carathéodory metric on $\partial \Omega$ (cf. [G2]) as follows. Call a piecewise $C^{1}$-smooth curve $\alpha:[0,1] \rightarrow \partial \Omega$ horizontal, if for $t \in[0,1]$ for which $\dot{\alpha}(t)$ exists we have $\dot{\alpha}(t) \in H_{\alpha(t)} \partial \Omega$. It follows from the strict pseudoconvexity of $\Omega$ that $\partial \Omega$ is connected. Moreover, any two points $p, q \in \partial \Omega$ can be joined by a horizontal curve $\alpha$ as follows from the contact property of $H \partial \Omega$ (see Section 3 for details). Define the Levi length of a curve by $L_{\rho}$-length $(\alpha):=\int_{0}^{1} L_{\rho}(\alpha(t) ; \dot{\alpha}(t))^{1 / 2} d t$, and for $p, q \in \partial \Omega$ let

$$
\begin{aligned}
& d_{H}(p, q)=\inf \left\{L_{\rho}-\operatorname{length}(\alpha): \alpha:[0,1] \rightarrow \partial \Omega\right. \text { is a horizontal curve } \\
& \text { with } \alpha(0)=p, \alpha(1)=q\} .
\end{aligned}
$$

At each point $p \in \partial \Omega$ we consider the splitting $\mathbf{C}^{n}=H_{p} \partial \Omega \oplus N_{p} \partial \Omega$ where $N_{p} \partial \Omega$ is the complex one-dimensional subspace of $\mathbf{C}^{n}$ orthogonal to $H_{p} \partial \Omega$. Accordingly, a vector $Z \in \mathbf{C}^{n}$ can uniquely be written as $Z=Z_{H}+Z_{N}$, where $Z_{H} \in H_{p} \partial \Omega$ and $Z_{N} \in N_{p} \partial \Omega$. In our notation we suppress the dependence on $p$.

For a horizontal curve $\alpha:[0,1] \rightarrow \partial \Omega$ we have that $\dot{\alpha}_{N} \equiv 0$ (in the points of differentiability) and so length $(\alpha)=\int_{0}^{1}\left|\dot{\alpha}_{H}(t)\right| d t$. The strict pseudoconvexity of 
$\Omega$ implies that there exists a constant $C \geq 1$ such that

$$
(1 / C)|Z| \leq L_{\rho}(p ; Z)^{1 / 2} \leq C|Z| \quad \text { for } \quad p \in \partial \Omega, Z \in H_{p} \partial \Omega
$$

So if we replace the Levi length of a curve by its Euclidean length in the definition of the Carnot-Carathéodory metric, then we get a bilipschitzly equivalent metric.

For each $x \in \Omega$ choose a point $\pi(x) \in \partial \Omega$ with $|x-\pi(x)|=\delta(x)$. This gives us a map $\pi: \Omega \rightarrow \partial \Omega$. Since $\Omega$ has a $C^{2}$-smooth boundary, the point $\pi(x) \in \partial \Omega$ with $|x-\pi(x)|=\delta(x)$ is uniquely determined if $x$ is sufficiently close to the boundary. We introduce the function $g: \Omega \times \Omega \rightarrow \mathbf{R}$ by

$$
g(x, y)=2 \log \left[\frac{d_{H}(\pi(x), \pi(y))+h(x) \vee h(y)}{\sqrt{h(x) h(y)}}\right]
$$

where the "height" $h$ is $h(x):=\delta(x)^{1 / 2}$ for $x \in \Omega, a \vee b:=\max \{a, b\}$, and $d_{H}$ is the Carnot-Carathéodory metric on $\partial \Omega$.

There is a certain ambiguity in the definition of $g$ due to the fact that a map $\pi$ with the required properties is not uniquely determined on the whole domain $\Omega$, but only on a sufficiently small neighborhood of $\partial \Omega$. Different choices of $\pi$ lead to functions in (1.2) that agree up to a bounded additive term. This will not affect the results below.

The formula (1.2) has its origins in the general framework of Gromov hyperbolic spaces (cf. Section 5). There are various similar expressions that serve the same purpose as $g$ in Theorem 1.1 below. The expression $g$ has the advantage that it is a pseudometric on the domain $\Omega$ and even a metric if we restrict it to a sufficiently small neighborhood of $\partial \Omega$.

A Finsler metric on $\Omega$ is a continuous map $F: \Omega \times \mathbf{C}^{n} \rightarrow \mathbf{R}_{+}:=[0, \infty)$ such that $F(x ; t Z)=|t| F(x ; Z)$ for all $x \in \Omega, t \in \mathbf{C}, Z \in \mathbf{C}^{n}$. The distance function $d_{F}$ associated with $F$ is defined by

$$
\begin{array}{r}
d_{F}(x, y)=\inf \left\{F \text {-length }(\gamma): \gamma:[0,1] \rightarrow \Omega \text { is a piecewise } C^{1}\right. \text {-smooth curve } \\
\text { with } \gamma(0)=x, \gamma(1)=y\},
\end{array}
$$

where

$$
F \text {-length }(\gamma)=\int_{0}^{1} F(\gamma(t) ; \dot{\gamma}(t)) d t
$$

Our main result shows how a certain local estimate for a Finsler metric leads to global estimates for the associated distance function.

Theorem 1.1. Let $\Omega \subseteq \mathbf{C}^{n}, n \geq 2$, be a bounded, strictly pseudoconvex domain with $C^{2}$-smooth boundary. Suppose $F$ is a Finsler metric on $\Omega$ with the following property. There exist constants $\epsilon_{0}>0, s>0, C_{1}>0, C_{2} \geq 1$ such that for all 
$x \in N_{\epsilon_{0}}(\partial \Omega) \cap \Omega$ and all $Z \in \mathbf{C}^{n}$ we have

$$
\begin{aligned}
\left(1-C_{1} \delta^{s}(x)\right) & \left(\frac{\left|Z_{N}\right|^{2}}{4 \delta^{2}(x)}+\left(1 / C_{2}\right) \frac{L_{\rho}\left(\pi(x) ; Z_{H}\right)}{\delta(x)}\right)^{1 / 2} \leq F(x ; Z) \\
& \leq\left(1+C_{1} \delta^{s}(x)\right)\left(\frac{\left|Z_{N}\right|^{2}}{4 \delta^{2}(x)}+C_{2} \frac{L_{\rho}\left(\pi(x) ; Z_{H}\right)}{\delta(x)}\right)^{1 / 2} .
\end{aligned}
$$

If $d_{F}$ is the distance function associated with $F$ and $g$ is defined as in (1.2), then there exists a constant $C \geq 0$ such that for all $x, y \in \Omega$

$$
g(x, y)-C \leq d_{F}(x, y) \leq g(x, y)+C .
$$

In (1.3) (and in (1.4) below) the splitting $Z=Z_{N}+Z_{H}$ is understood to be taken at $p=\pi(x)$.

Let $\mathbf{D}$ be the unit disc in $\mathbf{C}$. If $f: \mathbf{D} \rightarrow \Omega$ is a holomorphic map we denote by $D f(z)$ its differential mapping at the point $z \in \mathbf{D}$. The Kobayashi metric on $\Omega$ is a differential metric defined for $x \in \Omega$ and $Z \in \mathbf{C}^{n}$ by

$K(x ; Z)=\inf \{|v|: v \in \mathbf{C}$ and there exists a holomorphic map

$$
f: \mathbf{D} \rightarrow \Omega \text { with } f(0)=x \text { and } D f(0) v=Z\} .
$$

The Kobayashi distance $d_{K}$ is the distance function associated with the Kobayashi metric $K$.

In order to apply Theorem 1.1 to the Kobayashi metric we need an estimate as in (1.3). This type of estimate is given in the following proposition. This result cannot explicitly be found in the literature, but it can be obtained from slightly modifying the argument of in [M3] (cf. Section 4).

Proposition 1.2. Let $\Omega \subseteq \mathbf{C}^{n}, n \geq 2$, be a bounded, strictly pseudoconvex domain with $C^{2}$-smooth boundary. If $K$ is the Kobayashi metric on $\Omega$, then for every $\epsilon>0$, there exists $\epsilon_{0}>0$ and $C \geq 0$ such that for all $x \in N_{\epsilon_{0}}(\partial \Omega) \cap \Omega$ and all $Z \in \mathbf{C}^{n}$ we have

$$
\begin{aligned}
\left(1-C \delta^{1 / 2}(x)\right) & \left(\frac{\left|Z_{N}\right|^{2}}{4 \delta^{2}(x)}+(1-\epsilon) \frac{L_{\rho}\left(\pi(x) ; Z_{H}\right)}{\delta(x)}\right)^{1 / 2} \leq K(x ; Z) \\
& \leq\left(1+C \delta^{1 / 2}(x)\right)\left(\frac{\left|Z_{N}\right|^{2}}{4 \delta^{2}(x)}+(1+\epsilon) \frac{L_{\rho}\left(\pi(x) ; Z_{H}\right)}{\delta(x)}\right)^{1 / 2}
\end{aligned}
$$

It is important to notice that the magnitude of the vectors in the (complex) normal direction is quadratically bigger than the magnitude of horizontal vectors. 
(This is illustrated by the scaling factors $1 / \delta^{2}$ versus $1 / \delta$.) From this respect, it is essential that (1.4) gives a more precise estimate in the normal direction than in the horizontal direction. This is the advantage of Proposition 1.2 in comparison to the results in [M1], [M2], [M3]. Let us also note that due to a different normalization of the defining function, our Levi form $L_{\rho}$ differs by a factor 2 from the Levi form used in these papers.

Together with the previous theorem Proposition 1.2 gives the following corollary.

Corollary 1.3. Let $\Omega \subseteq \mathbf{C}^{n}, n \geq 2$, be a bounded, strictly pseudoconvex domain with $C^{2}$-smooth boundary. If $d_{K}$ is the Kobayashi distance on $\Omega$, then there exists a constant $C \geq 0$ such that for all $x, y \in \Omega$

$$
g(x, y)-C \leq d_{K}(x, y) \leq g(x, y)+C
$$

The statement and proof of the Theorem 1.1 have been motivated by the theory of Gromov hyperbolic spaces [BS], [GH], [G1]. In return, Corollary 1.3 implies the Gromov hyperbolicity of strictly pseudoconvex domains when equipped with the Kobayashi distance. This is formulated as follows.

Theorem 1.4. Let $\Omega \subseteq \mathbf{C}^{n}, n \geq 2$, be a bounded, strictly pseudoconvex domain with $C^{2}$-smooth boundary. If $d_{K}$ is the Kobayashi distance on $\Omega$, then the metric space $\left(\Omega, d_{K}\right)$ is hyperbolic in the sense of Gromov. The boundary $\partial_{G} \Omega$ of $\left(\Omega, d_{K}\right)$ as a Gromov hyperbolic space can be identified with the Euclidean boundary $\partial \Omega$. The Carnot-Carathéodory metric $d_{H}$ on $\partial \Omega$ lies in (and thus determines) the canonical class of snowflake equivalent metrics on $\partial_{G} \Omega$.

In the sequel (Section 5) we shall recall the notions and facts from the theory of Gromov hyperbolic spaces that are necessary for the proof of this theorem.

Theorem 1.4 implies that one can apply the general facts from the theory of Gromov hyperbolic spaces to strictly pseudoconvex domains and their maps. It follows that maps with certain nice properties on the domain can be extended to the boundary and vice versa. This gives a general framework for the classical regularity theory (cf. [Fef], [FR]) of extensions for biholomorphisms and proper holomorphic maps. In this spirit, we can deduce from Corollary 1.3 the well-known result that a proper holomorphic map between strictly pseudoconvex domains extends continuously to the closure of the domains. Actually, this map restricted to the boundaries will be Lipschitz in the Carnot-Carathéodory metrics (cf. Section 6 for precise statements).

The paper is organized as follows. Section 2 contains preparations for the proof of Theorem 1.1. In Section 3 we will discuss the Carnot-Carathéodory metric and prove a lemma that shows how to obtain this metric in a limiting sense from a class of Riemannian metrics. The proof of Theorem 1.1 is given in Section 4 
where we also indicate how to prove Proposition 1.2. In Section 5 we recall some background on Gromov hyperbolic spaces and give the proof of Theorem 1.4. We also show here that product-type domains are not Gromov hyperbolic. In the last section we present applications of Corollary 1.3 and Theorem 1.4 concerning boundary extension of mappings between strictly pseudoconvex domains. Finally, we relate our results to previously known estimates and discuss further possible developments.

The results of this paper for the Kobayashi metric have been announced in a slightly weaker form in $[\mathrm{BB}]$.

\section{Preliminaries: Preparations for the proof of Theorem 1.1}

Our first statement holds for domains in $\mathbf{R}^{n}, n \geq 2$. We denote by $B(x, r)$ the open Euclidean ball with radius $r>0$ centered at $x$ and by $\bar{B}(x, r)$ its closure. We denote the standard Euclidean scalar product of two vectors $a, b \in \mathbf{R}^{n}$ by $(a \cdot b)$. Moreover, $[a, b]$ will be the closed segment in $\mathbf{R}^{n}$ with endpoints $a, b \in \mathbf{R}^{n}$, and we will use the obvious notation for the open and half-open segments as well.

Lemma 2.1. Suppose that $\Omega \subseteq \mathbf{R}^{n}, n \geq 2$, is a bounded domain with $C^{2}$-smooth boundary. Let $\delta(x)=\operatorname{dist}(x, \partial \Omega)$ for $x \in \mathbf{R}^{n}$. Then there exists $\epsilon_{0}>0$ such that

(a) for every point $x \in N_{\epsilon_{0}}(\partial \Omega)$ there exists a unique point $\pi(x) \in \partial \Omega$ with $|x-\pi(x)|=\delta(x)$,

(b) the signed distance to the boundary $\rho: \mathbf{R}^{n} \rightarrow \mathbf{R}$ is $C^{2}$-smooth on $N_{\epsilon_{0}}(\partial \Omega)$,

(c) for the fibers of the map $\pi: N_{\epsilon_{0}}(\partial \Omega) \rightarrow \partial \Omega$ we have

$$
\pi^{-1}(p)=S_{p}:=\left(p-\epsilon_{0} n(p), p+\epsilon_{0} n(p)\right),
$$

where $n(p)$ is the outer unit normal vector of $\partial \Omega$ at $p \in \partial \Omega$,

(d) the gradient of $\rho$ satisfies

$$
\operatorname{grad} \rho(x)=n(\pi(x)) \quad \text { for all } \quad x \in N_{\epsilon_{0}}(\partial \Omega),
$$

(e) the projection map $\pi: N_{\epsilon_{0}}(\partial \Omega) \rightarrow \partial \Omega$ is $C^{1}$-smooth.

Proof. (a) The proof of this statement is due to Federer [Fed].

(b) This was proved in [KP]. The proof is based on (a).

(c) Let $p \in \partial \Omega$ and consider the ball $B(x,|x-p|)$, where $x$ lies on the segment $S_{p}=\left(p-\epsilon_{0} n(p), p+\epsilon_{0} n(p)\right)$. This ball is tangent to $\partial \Omega$, and it is easy to see that for $x \in S_{p}$ close to $p$ we have $\bar{B}(x,|x-p|) \cap \partial \Omega=\{p\}$. We show that this is true for all $x \in S_{p}$. Otherwise, there would be a first $x_{0} \in S_{p}$ (as we move along $S_{p}$ in one of the directions away from $p)$ for which $\bar{B}\left(x_{0},\left|x_{0}-p\right|\right) \cap \partial \Omega \neq\{p\}$. Then there exists a point $p^{\prime} \in \partial \Omega, p^{\prime} \neq p$, such that $\left\{p, p^{\prime}\right\} \subseteq \bar{B}\left(x_{0},\left|x_{0}-p\right|\right) \cap \partial \Omega \subseteq \partial B\left(x_{0},\left|x_{0}-p\right|\right)$. In particular, $\left|p-x_{0}\right|=\left|p^{\prime}-x_{0}\right|=\delta\left(x_{0}\right)$ contradicting (a). This shows that 
$S_{p} \subseteq \pi^{-1}(p)$. Conversely, if $x \in \pi^{-1}(p), x \neq p$, then $[x, p] \subseteq \pi^{-1}(p)$ and it is easy to see that $x-p$ is normal to $\partial \Omega$. Since $|x-p|<\epsilon_{0}$ it follows that $x \in S_{p}$. In conclusion we have

$$
N_{\epsilon_{0}}(\partial \Omega)=\pi^{-1}(\partial \Omega)=\bigcup_{p \in \partial \Omega} \pi^{-1}(p)=\bigcup_{p \in \partial \Omega} S_{p}
$$

(d) Let us first notice that $|\rho(x)-\rho(y)| \leq|x-y|$ for $x, y \in N_{\epsilon_{0}}(\partial \Omega)$. This implies that $|\operatorname{grad} \rho(x)| \leq 1$ for $x \in N_{\epsilon_{0}}(\partial \Omega)$.

Choose a point $p \in \partial \Omega$ and let $x=p+t_{0} n(p)$ for some fixed $t_{0} \in\left(-\epsilon_{0}, \epsilon_{0}\right)$. Consider the points $x_{t}=p+\left(t_{0}+t\right) n(p)$ for $t>0$ small enough such that $x_{t} \in N_{\epsilon_{0}}(\partial \Omega)$. Then $x_{t}, x \in \pi^{-1}(p)$ by (c) and therefore

$$
\rho\left(x_{t}\right)-\rho(x)=|| x_{t}-p|-| x-p||=t .
$$

Using Taylor's expansion we have

$$
t=\rho\left(x_{t}\right)-\rho(x)=(\operatorname{grad} \rho(x) \cdot n(p)) t+o(t) .
$$

Since $|\operatorname{grad} \rho(x)| \leq 1$ this implies that $\operatorname{grad} \rho(x)=n(p)$.

(e) Let $x \in N_{\epsilon_{0}}(\partial \Omega)$, and $p=\pi(x)$. Then (c) implies that $x=p+\rho(x) n(p)$. On the other hand, $n(p)=\operatorname{grad} \rho(x)$ by (d) and thus

$$
\pi(x)=p=x-\rho(x) \operatorname{grad} \rho(x) .
$$

From this expression for $\pi$ and (b) it follows that $\pi$ is a $C^{1}$-map.

In what follows we shall consider bounded domains $\Omega \subseteq \mathbf{C}^{n}, n \geq 2$, with $C^{2}$-smooth boundary. If we identify $\mathbf{C}^{n}$ with $\mathbf{R}^{2 n}$ so that $\left(Z_{1}, \ldots, Z_{n}\right) \in \mathbf{C}^{n}$ corresponds to $\left(\operatorname{Re} Z_{1}, \operatorname{Im} Z_{1}, \ldots, \operatorname{Re} Z_{n}, \operatorname{Im} Z_{n}\right) \in \mathbf{R}^{2 n}$, then we can use the results of the previous lemma for these domains. Note that under the above identification $n:=2 \bar{\partial} \rho$ corresponds to $\operatorname{grad} \rho$. So $n(p)$ is the outer unit normal to $\partial \Omega$ for $p \in \partial \Omega$.

Recall that for any $p \in \partial \Omega$ vectors $Z \in \mathbf{C}^{n}$ can be written as $Z=Z_{H}+Z_{N}$ in a unique way such that $Z_{H} \in H_{p} \partial \Omega$ and $Z_{N} \in N_{p} \partial \Omega$. In the following lemma we shall relate curves $\gamma:[0,1] \rightarrow N_{\epsilon_{0}}(\partial \Omega) \cap \Omega$ and their projections $\alpha=\pi \circ \gamma$, where $\epsilon_{0}>0$ is a sufficiently small constant. For the tangent vectors $\dot{\gamma}(t)$ and $\dot{\alpha}(t)$ of these curves we will consider the splitting into horizontal and normal parts at $\alpha(t)=\pi \circ \gamma(t)$ and write this as $\dot{\gamma}(t)=\dot{\gamma}_{H}(t)+\dot{\gamma}_{N}(t)$ and $\dot{\alpha}(t)=\dot{\alpha}_{H}(t)+\dot{\alpha}_{N}(t)$.

Lemma 2.2. Let $\Omega \subseteq \mathbf{C}^{n}, n \geq 2$, be a bounded domain with $C^{2}$-smooth boundary. Then there exist constants $\epsilon_{0}>0$ and $C>0$ with the following property. If $\gamma:[0,1] \rightarrow N_{\epsilon_{0}}(\partial \Omega) \cap \Omega$ is a $C^{1}$-smooth curve and $\alpha=\pi \circ \gamma$ its projection to the boundary, then the following estimates hold for $t \in[0,1]$

(2.2) $\left|\dot{\gamma}_{H}(t)-\dot{\alpha}_{H}(t)\right| \leq C \delta(\gamma(t))|\dot{\gamma}(t)|$, 
(2.3) $\left|\dot{\gamma}_{H}(t)-\dot{\alpha}_{H}(t)\right| \leq C \delta(\gamma(t))|\dot{\alpha}(t)|$,

(2.4) $\left|\dot{\alpha}_{N}(t)\right| \leq\left|\dot{\gamma}_{N}(t)\right|+C \delta(\gamma(t))|\dot{\gamma}(t)|$,

(2.5) $\left|\dot{\gamma}_{N}(t)\right| \leq\left|\dot{\alpha}_{N}(t)\right|+C \delta_{0}|\dot{\alpha}(t)|$ if $\delta(\gamma(t)) \equiv \delta_{0}$.

Proof. Choose $\epsilon_{0}>0$ sufficiently small so that we can apply the statements in Lemma 2.1. In addition, we may assume that all derivatives of the defining function $\rho$ up to second order are uniformly bounded on $N_{\epsilon_{0}}(\partial \Omega)$. By Lemma 2.1 (e), the curve $\alpha$ is $C^{1}$-smooth, and so $\dot{\alpha}$ is defined. From (2.1) we get the following relation

$$
\alpha(t)-\gamma(t)=-\rho(\gamma(t)) n(\gamma(t))=-\rho(\gamma(t)) n(\alpha(t)),
$$

where $n(x)=2 \bar{\partial} \rho(x)$. Note that $n(x)=n(\pi(x))$ and $n(x)_{H}=0$ at $\pi(x)$ for $x \in N_{\epsilon_{0}}(\partial \Omega)$ (cf. Lemma 2.1 (c) and (d)).

Differentiating the equalities in (2.6) we obtain

$$
\dot{\alpha}(t)-\dot{\gamma}(t)=\rho(\gamma(t))\left[M_{1}(t) \dot{\gamma}(t)+M_{2}(t) \overline{\dot{\gamma}(t)}\right]+\operatorname{Re}\langle a(t), \dot{\gamma}(t)\rangle n(\gamma(t)),
$$

and

$$
\dot{\alpha}(t)-\dot{\gamma}(t)=\rho(\gamma(t))\left[M_{3}(t) \dot{\alpha}(t)+M_{4}(t) \overline{\dot{\alpha}(t)}\right]+\operatorname{Re}\langle a(t), \dot{\gamma}(t)\rangle n(\alpha(t)),
$$

where $M_{1}, \ldots, M_{4}:[0,1] \rightarrow M_{n}(\mathbf{C})$ are complex $(n \times n)$-matrix valued functions, and $a:[0,1] \rightarrow \mathbf{C}^{n}$ is a complex vector valued function. The components of these functions can be expressed by the first and second derivatives of $\rho$ evaluated at points in $N_{\epsilon_{0}}(\partial \Omega)$. Thus these components are uniformly bounded independently of $\gamma$. Taking the projection onto the horizontal subspace (at $\alpha(t))$ in $(2.7)$, relation (2.2) follows. Similarly, (2.3) follows from (2.8). To prove (2.4) observe first that $\alpha(t) \subseteq \partial \Omega$ for $t \in[0,1]$ gives $\operatorname{Re}\langle\dot{\alpha}(t), n(\alpha(t))\rangle=0$. Thus

$$
\left|\dot{\alpha}_{N}(t)\right|=|\operatorname{Im}\langle\dot{\alpha}(t), n(\alpha(t))\rangle| .
$$

Taking the hermitian product with $n(\gamma(t))$ in (2.7) and using (2.9), we get (2.4). To show (2.5) we differentiate (2.6) under the condition $\rho(\gamma(t))=-\delta_{0}$. This gives

$$
\dot{\alpha}(t)-\dot{\gamma}(t) \equiv-\delta_{0}\left[M_{3}(t) \dot{\alpha}(t)+M_{4}(t) \overline{\dot{\alpha}(t)}\right],
$$

which implies (2.5) by taking again the normal projection.

Let us mention that if $\delta(\gamma(t)) \equiv \delta_{0}$ and $\alpha$ is a horizontal curve, i.e., $\dot{\alpha}_{N} \equiv 0$, then (2.5) takes the form

$$
\left|\dot{\gamma}_{N}(t)\right| \leq C \delta_{0}|\dot{\alpha}(t)|
$$

An essential ingredient in the proof of Theorem 1.1 is that the Carnot-Cara théodory metric on $\partial \Omega$ can be approximated by a class of Riemannian metrics. 
This is stated as the Approximation Lemma (Lemma 3.2) in the next section. The following lemma will facilitate the use of the Approximation Lemma in the proof of Theorem 1.1.

Lemma 2.3. Let $\Omega \subseteq \mathbf{C}^{n}, n \geq 2$, be a bounded strictly pseudoconvex domain with $C^{2}$-smooth boundary. Then there exists $\epsilon_{0}>0$ and $C>0$ with the following property. If $\gamma:[0,1] \rightarrow N_{\epsilon_{0}}(\partial \Omega) \cap \Omega$ is $C^{1}$-smooth and $\alpha=\pi \circ \gamma$, then for $t \in[0,1]$ we have

$$
\frac{L_{\rho}\left(\pi(\gamma(t)) ; \dot{\gamma}_{H}(t)\right)}{\delta(\gamma(t))}+\frac{\left|\dot{\gamma}_{N}(t)\right|^{2}}{4 \delta(\gamma(t))^{2}} \geq C\left[\frac{L_{\rho}\left(\alpha(t) ; \dot{\alpha}_{H}(t)\right)}{\delta(\gamma(t))}+\frac{\left|\dot{\alpha}_{N}(t)\right|^{2}}{\delta(\gamma(t))^{2}}\right]
$$

Proof. In the course of the proof we will see how to choose the number $\epsilon_{0}>0$, but we may assume that it is small enough so that we can use lemmas 2.1 and 2.2. From $|Z|^{2} \geq \frac{1}{2}|W|^{2}-|Z-W|^{2}$ for $Z, W \in \mathbf{C}^{n}$ and inequalities (2.2) and (2.4) we obtain

$$
\left|\dot{\gamma}_{H}(t)\right|^{2} \geq \frac{1}{2}\left|\dot{\alpha}_{H}(t)\right|^{2}-C_{1} \delta(\gamma(t))^{2}|\dot{\gamma}(t)|^{2}
$$

and

$$
\left|\dot{\gamma}_{N}(t)\right|^{2} \geq \frac{1}{2}\left|\dot{\alpha}_{N}(t)\right|^{2}-C_{2} \delta(\gamma(t))^{2}|\dot{\gamma}(t)|^{2}
$$

Here and in the following $C_{1}, C_{2}, \ldots$ are positive constants independent of $\gamma$ and $t$. Let us denote the left hand side of (2.11) by $A$. Then the relations (1.1), (2.12), and (2.13) imply

$$
A / 2 \geq C_{3}\left(\frac{\left|\dot{\alpha}_{H}(t)\right|^{2}}{\delta(\gamma(t))}+\frac{\left|\dot{\alpha}_{N}(t)\right|^{2}}{\delta(\gamma(t))^{2}}\right)-C_{4}|\dot{\gamma}(t)|^{2}
$$

If $\epsilon_{0}>0$ is small enough we will always have $A / 2 \geq C_{4}|\dot{\gamma}(t)|^{2}$ by (1.1). Thus

$$
\begin{aligned}
A & \geq C_{3}\left(\frac{\left|\dot{\alpha}_{H}(t)\right|^{2}}{\delta(\gamma(t))}+\frac{\left|\dot{\alpha}_{N}(t)\right|^{2}}{\delta(\gamma(t))^{2}}\right) \\
& \geq C_{5}\left(\frac{L_{\rho}\left(\alpha(t) ; \dot{\alpha}_{H}(t)\right)}{\delta(\gamma(t))}+\frac{\left|\dot{\alpha}_{N}(t)\right|^{2}}{\delta(\gamma(t))^{2}}\right) .
\end{aligned}
$$

\section{The Carnot-Carathéodory metric}

In this section we shall deal with the horizontal or Carnot-Carathéodory metric $d_{H}$ on $\partial \Omega$ defined in the introduction. A recent account on the subject can be found in $[\mathrm{Be}]$ and $[\mathrm{G} 2]$. 
In the present setting of strictly pseudoconvex boundaries the definition of this metric requires additional explanation. Let us briefly indicate why two points can be connected by a horizontal curve. We notice first that $\partial \Omega$ is connected. Indeed, the strict pseudoconvexity of $\Omega$ implies that $H_{c}^{n-1}(\Omega)=\{0\}$ which in turn implies that $\partial \Omega$ is connected if $n \geq 2$. Furthermore, it also follows from the strict pseudoconvexity of $\Omega$ that $H \partial \Omega$ is a contact bundle. Therefore, vector fields in $H \partial \Omega$ generate the whole tangent bundle $T \partial \Omega$. A theorem due to Chow (cf. [Be, p. 15]) shows that any two points in $\partial \Omega$ can be connected by a $C^{1}$-smooth horizontal curve. Thus $d_{H}$ is well-defined and bounded. In our setting the size of balls can be described quite explicitly by the following proposition.

Proposition 3.1. (Box-Ball estimate) Suppose $\Omega \subseteq \mathbf{C}^{n}, n \geq 2$, is a bounded strictly pseudoconvex domain with $C^{2}$-smooth boundary. Then there exists $\epsilon_{0}>0$ and $C \geq 1$ such that for all $0<\epsilon \leq \epsilon_{0}$ and $p \in \partial \Omega$

$$
\operatorname{Box}(p, \epsilon / C) \subseteq B_{H}(p, \epsilon) \subseteq \operatorname{Box}(p, C \epsilon),
$$

where $B_{H}(p, \epsilon)=\left\{q \in \partial \Omega: d_{H}(p, q)<\epsilon\right\}$ and $\operatorname{Box}(p, \epsilon)=\left\{p+Z \in \partial \Omega:\left|Z_{H}\right|<\right.$ $\left.\epsilon,\left|Z_{N}\right|<\epsilon^{2}\right\}$. Here the decomposition $Z=Z_{H}+Z_{N}$ is taken at $p$.

We will not give the proof of this proposition, because its content is wellknown. Indeed, much more general statements are true (cf. [NSW], [Be], [G2]). For the case of the Heisenberg group a more direct proof is given in Sect. 3 and Sect. 4 of [Ko]. Since boundaries of strictly pseudoconvex domains can be locally approximated by the Heisenberg group in a quantitative sense (Sect. 14 of [FS]), our present statement can be derived from this.

It follows from Proposition 3.1 that there exist constants $C_{1}, C_{2}>0$ such that

$$
C_{1}|p-q| \leq d_{H}(p, q) \leq C_{2}|p-q|^{1 / 2} \quad \text { for } \quad p, q \in \partial \Omega .
$$

In particular, the topology on $\partial \Omega$ induced by the Carnot-Carathéodory metric agrees with the topology induced by the Euclidean metric.

The essential ingredient used in the proof of Theorem 1.1 is that the CarnotCarathéodory metric can be approximated by a class of Riemannian metrics $G_{\kappa}$ on $\partial \Omega$. To be specific, fix $\kappa>0$, and for $p \in \partial \Omega, Z \in T_{p} \partial \Omega$ let

$$
G_{\kappa}^{2}(p ; Z)=L_{\rho}\left(p ; Z_{H}\right)+\kappa^{2}\left|Z_{N}\right|^{2} .
$$

The distance function $d_{\kappa}$ associated with this Riemannian metric approximates the Carnot-Carathéodory metric $d_{H}$ in the following quantitative sense.

Lemma 3.2. (Approximation Lemma) There is a constant $C>0$ such that for all $\kappa>0$ the following holds. If $p, q \in \partial \Omega$ satisfy $d_{H}(p, q) \geq 1 / \kappa$, then

$$
\frac{1}{C} d_{\kappa}(p, q) \leq d_{H}(p, q) \leq C d_{\kappa}(p, q) .
$$


Similar statements can be found in [G2]. However, we will give a complete proof based on Proposition 3.1, since the Approximation Lemma is crucial in the proof of Theorem 1.1.

Proof. Let $\kappa>0$ be given and consider the Riemannian metric $G_{\kappa}$ as defined above. Let $p, q \in \partial \Omega$. For any piecewise $C^{1}$-smooth horizontal curve $\alpha$ in $\partial \Omega$ connecting $p$ and $q$ we have $\dot{\alpha}_{N} \equiv 0$ a.e. and therefore $G_{\kappa}(\alpha(t) ; \dot{\alpha}(t))^{2}=L_{\rho}(\alpha(t) ; \dot{\alpha}(t))$ for a.e. $t$. It follows that $G_{\kappa}$-length $(\alpha)=L_{\rho}$-length $(\alpha)$ for horizontal curves $\alpha$. For the value of $d_{\kappa}(p, q)$ we minimize the $G_{\kappa}$-length over all, not just the horizontal curves joining $p$ and $q$. Hence

$$
d_{\kappa}(p, q) \leq d_{H}(p, q) .
$$

In order to obtain a lower bound for $d_{\kappa}(p, q)$ we will prove that there exist constants $\kappa_{0}>0$ and $C>0$ such that we have the implication

$$
d_{H}(a, b) \geq 1 / \kappa \Rightarrow d_{\kappa}(a, b) \geq C / \kappa \quad \text { whenever } \quad a, b \in \partial \Omega, \kappa \geq \kappa_{0} .
$$

Let us suppose that (3.3) holds and let $p, q \in \partial \Omega$ with $d_{H}(p, q) \geq 1 / \kappa$ be given. If $\kappa \geq \kappa_{0}$, let $\alpha:[0,1] \rightarrow \partial \Omega$ be a piecewise $C^{1}$-smooth curve with $\alpha(0)=p$ and $\alpha(1)=q$. There exist $N \in \mathbf{N}$ and $0=t_{0}<t_{1}<\cdots<t_{N}=1$ such that for $x_{j}=\alpha\left(t_{j}\right) \in \partial \Omega$ we have

$$
1 / \kappa \leq d_{H}\left(x_{j-1}, x_{j}\right) \leq 2 / \kappa, \quad j=1, \ldots, N .
$$

Then (3.3) applied to $x_{j-1}$ and $x_{j}$ leads to

$$
\begin{aligned}
G_{\kappa} \text {-length }(\alpha) & =\sum_{j=1}^{N} G_{\kappa} \text {-length }\left(\alpha \mid\left[t_{j-1}, t_{j}\right]\right) \geq \sum_{j=1}^{N} d_{\kappa}\left(x_{j-1}, x_{j}\right) \\
& \geq C N / \kappa \geq \frac{C}{2} \sum_{j=1}^{N} d_{H}\left(x_{j-1}, x_{j}\right) \geq \frac{C}{2} d_{H}(p, q) .
\end{aligned}
$$

Taking the infimum over all admissible curves $\alpha$ we obtain

$$
d_{\kappa}(p, q) \geq \frac{C}{2} d_{H}(p, q)
$$

If $0<\kappa<\kappa_{0}$, let $\kappa_{1}:=d_{H}$-diam $(\partial \Omega)^{-1}>0$. Note that $1 / \kappa \leq d_{H}(p, q) \leq$ $d_{H-\operatorname{diam}}(\partial \Omega)=1 / \kappa_{1}$ and so $\kappa \geq \kappa_{1}$. Since $G_{\kappa} \geq\left(\kappa / \kappa_{0}\right)^{2} G_{\kappa_{0}}$ we have $d_{\kappa} \geq$ $\left(\kappa / \kappa_{0}\right) d_{\kappa_{0}}$. Hence (3.3) and the definition of $\kappa_{1}$ give

$$
d_{\kappa}(p, q) \geq\left(\kappa_{1} / \kappa_{0}\right) d_{\kappa_{0}}(p, q) \geq C\left(\kappa_{1} / \kappa_{0}^{2}\right) \geq C\left(\kappa_{1} / \kappa_{0}\right)^{2} d_{H}(p, q) .
$$


The inequalities (3.4a) and (3.4b) are the lower estimates we want.

It remains to prove implication (3.3). In the following $C_{1}, C_{2}, \ldots$ will be constants only depending on $\Omega$, but not on $\kappa$ in particular. By the strict pseudoconvexity of $\Omega$ (cf. (1.1)) there exists constants $C_{1}, C_{2}>0$ such that

$$
C_{1}|Z|^{2} \leq L_{\rho}(p ; Z) \leq C_{2}|Z|^{2} \quad \text { for } \quad p \in \partial \Omega, Z \in H_{p} \partial \Omega .
$$

Let $\kappa_{0}=\max \left\{\sqrt{2 C_{1}}, 1 / \epsilon_{0}\right\}$ where $\epsilon_{0}$ is the constant in Proposition 3.1.

Suppose $\kappa \geq \kappa_{0}$ and let $a, b \in \partial \Omega$ with $d_{H}(a, b) \geq 1 / \kappa$ be given. Since $1 / \kappa \leq$ $\epsilon_{0}$, Proposition 3.1 implies that for some $0<C_{3} \leq 1$ we have $\operatorname{Box}\left(a, C_{3} / \kappa\right) \subseteq$ $B_{H}(a, 1 / \kappa)$, and so

$$
b \notin \operatorname{Box}\left(a, C_{3} / \kappa\right) .
$$

Since $\partial \Omega$ is $C^{2}$-smooth, the normal vector $n=2 \bar{\partial} \rho$ is $C^{1}$-smooth, and hence Lipschitz on $\partial \Omega$, i.e., there exists $C_{4}>0$ such that

$$
|n(x)-n(y)| \leq C_{4}|x-y| \quad \text { for } \quad x, y \in \partial \Omega .
$$

Now let $\alpha:[0,1] \rightarrow \partial \Omega$ be any piecewise $C^{1}$-smooth curve connecting $a$ and $b$, and let $A=\operatorname{length}(\alpha)$. Since $\dot{\alpha}(t) \in T_{\alpha(t)} \partial \Omega$ we have $\operatorname{Re}\langle\dot{\alpha}(t), n(\alpha(t))\rangle=0$ for a.e. $t \in[0,1]$. Therefore, (3.6) implies

$$
|\operatorname{Re}\langle\dot{\alpha}(t), n(a)\rangle| \leq C_{4} A|\dot{\alpha}(t)| \text {, for a.e. } t \in[0,1] .
$$

Integrating (3.7) we obtain

$$
|\operatorname{Re}\langle b-a, n(a)\rangle| \leq C_{4} A^{2}
$$

Furthermore, using $|Z|^{2} \geq \frac{1}{2}|W|^{2}-|Z-W|^{2}$ for $Z, W \in \mathbf{C}$ and (3.6) we get

$$
\begin{aligned}
\left|\dot{\alpha}_{N}(t)\right|^{2} & =|\langle\dot{\alpha}(t), n(\alpha(t))\rangle|^{2}=|\operatorname{Im}\langle\dot{\alpha}(t), n(\alpha(t))\rangle|^{2} \\
& \geq \frac{1}{2}|\operatorname{Im}\langle\dot{\alpha}(t), n(a)\rangle|^{2}-C_{4}^{2} A^{2}|\dot{\alpha}(t)|^{2} \text { for a.e. } t \in[0,1]
\end{aligned}
$$

Now we consider two cases according to whether $A \geq C_{5} / \kappa$ or not, where $C_{5}=$ $\min \left\{\frac{\sqrt{2 C_{1}}}{C_{4}}, \frac{C_{3}}{2 \sqrt{C_{4}}}, C_{3}\right\}$.

Recall that $\kappa \geq \kappa_{0} \geq \sqrt{2 C_{1}}$. If $A \geq C_{5} / \kappa$, then

$$
\begin{aligned}
G_{\kappa} \text {-length }(\alpha) & =\int_{0}^{1}\left(L_{\rho}\left(\alpha(t) ; \dot{\alpha}_{H}(t)\right)+\kappa^{2}\left|\dot{\alpha}_{N}(t)\right|^{2}\right)^{1 / 2} d t \\
& \geq \int_{0}^{1}\left(C_{1}\left|\dot{\alpha}_{H}(t)\right|^{2}+\kappa^{2}\left|\dot{\alpha}_{N}(t)\right|^{2}\right)^{1 / 2} d t \\
& \geq \sqrt{C_{1}} \operatorname{length}(\alpha)=\sqrt{C_{1}} A \geq\left(\sqrt{C_{1}} C_{5}\right) / \kappa .
\end{aligned}
$$


If $A<C_{5} / \kappa$, then because of $C_{5} \leq \sqrt{2 C_{1}} / C_{4}$ we have that $C_{1}-\frac{\kappa^{2}}{2} C_{4}^{2} A^{2} \geq 0$, and so by (3.9)

$$
\begin{aligned}
G_{\kappa} \text {-length }(\alpha) & \geq \int_{0}^{1}\left(C_{1}\left|\dot{\alpha}_{H}(t)\right|^{2}+\kappa^{2}\left|\dot{\alpha}_{N}(t)\right|^{2}\right)^{1 / 2} d t \\
& \geq \int_{0}^{1}\left(C_{1}|\dot{\alpha}(t)|^{2}+\frac{\kappa^{2}}{2}\left|\dot{\alpha}_{N}(t)\right|^{2}\right)^{1 / 2} d t \\
& \geq \frac{\kappa}{2} \int_{0}^{1}|\operatorname{Im}\langle\dot{\alpha}(t), n(a)\rangle| d t \geq \frac{\kappa}{2}|\operatorname{Im}\langle b-a, n(a)\rangle| .
\end{aligned}
$$

Using (3.8) and $C_{5} \leq \frac{C_{3}}{2 \sqrt{C_{4}}}$ we get

$$
\begin{aligned}
|\operatorname{Im}\langle b-a, n(a)\rangle| & \geq|\langle b-a, n(a)\rangle|-|\operatorname{Re}\langle b-a, n(a)\rangle| \\
& \geq\left|(b-a)_{N}\right|-C_{4} A^{2} \geq\left|(b-a)_{N}\right|-\frac{C_{3}^{2}}{4 \kappa^{2}},
\end{aligned}
$$

where $(b-a)_{N}$ is taken at $a$, i.e., stands for the projection of $(b-a)$ onto $N_{a} \partial \Omega$.

Relations (3.11) and (3.12) give

$$
G_{\kappa} \text {-length }(\alpha) \geq \frac{\kappa}{2}\left|(b-a)_{N}\right|-\frac{C_{3}^{2}}{8 \kappa} .
$$

On the other hand, $C_{5} \leq C_{3}$ implies

$$
|b-a| \leq \operatorname{length}(\alpha)=A<C_{3} / \kappa
$$

In view of (3.5) this shows that we must have $\left|(b-a)_{N}\right| \geq C_{3}^{2} / \kappa^{2}$. Consequently, by $(3.13)$

$$
G_{\kappa} \text {-length }(\alpha) \geq \frac{3 C_{3}^{2}}{8 \kappa}
$$

Relations (3.10) and (3.14) show that $G_{\kappa}$-length $(\alpha) \geq C / \kappa$ for a uniform $C>0$. Taking the infimum over $\alpha$ we get (3.3).

\section{Proof of Theorem 1.1 and Proposition 1.2}

Let us fix a small constant $\epsilon_{0}>0$. Define $K=\left\{x \in \Omega: \delta(x) \geq \epsilon_{0}\right\}$ and $N=\left\{x \in \Omega: \delta(x)<\epsilon_{0}\right\}=N_{\epsilon_{0}}(\partial \Omega) \cap \Omega$. Then $K$ is compact, $N$ is open, $N \cap K=\emptyset$, and $N \cup K=\Omega$. We assume that $\epsilon_{0}$ is so small that $\bar{N} \cap \Omega$ lies in a sufficiently small neighborhood of the boundary for which we can use the results of lemmas 2.1-2.3. Moreover, we assume that for $x \in \bar{N} \cap \Omega$ and $Z \in \mathbf{C}^{n}$ our given differential metric $F$ satisfies (1.3) for some constants $s>0, C_{1}>0$ and $C_{2} \geq 1$ and that $1-C_{1} \delta^{s}(x) \geq 1 / 2$. 
Recall that $d_{H}$ is the horizontal metric on $\partial \Omega, h(x)=\delta(x)^{1 / 2}$ is the height of a point $x \in \Omega$, and for $p \in \partial \Omega$ the vector $n(p)=2 \bar{\partial} \rho(p)$ is the outer normal to $\partial \Omega$ at $p$. In what follows we will denote by $C$ positive constants only depending on $\epsilon_{0}$ and the various other constants that are associated with $\Omega$ and $F$. The actual value of $C$ does not matter and may change even within the same line. Given our assumptions and our notation, we prove a lemma that we will use repeatedly in the following.

Lemma 4.1. Suppose $\gamma:[0,1] \rightarrow \bar{N} \cap \Omega$ is a piecewise $C^{1}$-smooth curve with endpoints $u=\gamma(0)$ and $v=\gamma(1)$. Then

$$
F-\operatorname{length}(\gamma) \geq\left|\log \left(\frac{h(v)}{h(u)}\right)\right|-C .
$$

If $\gamma:[0,1] \rightarrow \bar{N} \cap \Omega, t \mapsto u+t(v-u)$, is a straight line segment contained in some fiber $\pi^{-1}(p), p \in \partial \Omega$, then

$$
F \text { - length }(\gamma)=\left|\log \left(\frac{h(v)}{h(u)}\right)\right| \pm C \text {. }
$$

Here and in the following we write $A=B \pm C$ if $|A-B| \leq C$.

Proof. By Lemma 2.1 we have for those $t_{0} \in[0,1]$ for which $\dot{\gamma}\left(t_{0}\right)$ exists

$$
\begin{aligned}
\left|\frac{d}{d t} h(\gamma(t))\right|_{t=t_{0}} & =\frac{1}{2 h\left(\gamma\left(t_{0}\right)\right)}\left|\frac{d}{d t} \delta(\gamma(t))\right|_{t=t_{0}}=\frac{\left|\operatorname{Re}\left\langle\bar{\partial} \delta\left(\gamma\left(t_{0}\right)\right), \dot{\gamma}\left(t_{0}\right)\right\rangle\right|}{h\left(\gamma\left(t_{0}\right)\right)} \\
& =\frac{\left|\operatorname{Re}\left\langle n\left(\pi\left(\gamma\left(t_{0}\right)\right)\right), \dot{\gamma}\left(t_{0}\right)\right\rangle\right|}{2 h\left(\gamma\left(t_{0}\right)\right)} \leq \frac{\left|\dot{\gamma}_{N}\left(t_{0}\right)\right|}{2 h\left(\gamma\left(t_{0}\right)\right)} .
\end{aligned}
$$

By (1.3) this implies that

$$
\begin{aligned}
F \text {-length }(\gamma) & \geq \int_{0}^{1}\left(1-C_{1} h(\gamma(t))^{2 s}\right) \frac{\left|\dot{\gamma}_{N}(t)\right|}{2 h^{2}(\gamma(t))} d t \\
& \geq \int_{0}^{1} \mid d\left(\log \left(h(\gamma(t))-\frac{C_{1}}{2 s} h(\gamma(t))^{2 s}\right) \mid\right. \\
& \geq \mid\left[\log \left(h(\gamma(t))-\frac{C_{1}}{2 s} h(\gamma(t))^{2 s}\right]_{0}^{1} \mid\right. \\
& \geq\left|\log \left(\frac{h(v)}{h(u)}\right)\right|-C .
\end{aligned}
$$

If $\gamma \subseteq \bar{N} \cap \pi^{-1}(p)$ is a straight line segment, it follows from Lemma 2.1 that $\dot{\gamma}(t) \equiv$ $\pm|u-v| n(p)$ and $n(\pi(\gamma(t)))=n(p)$ for $t \in[0,1]$. Moreover, $\left|\dot{\gamma}_{N}(t)\right|=|u-v|$, and 
$\dot{\gamma}_{H}(t) \equiv 0$. Using this and the upper estimate for $F$ we obtain by a computation similar to the previous one

$$
F \text { - length }(\gamma) \leq\left|\log \left(\frac{h(v)}{h(u)}\right)\right|+C
$$

Proof of Theorem 1.1. We need to show that there exists a constant $C>0$ such that

$$
g(x, y)-C \leq d_{F}(x, y) \leq g(x, y)+C \quad \text { for } \quad x, y \in \Omega .
$$

In order to prove (4.1) we shall consider various cases depending on the relative position of $x$ and $y$. Some of the cases lead to well-known estimates (see e.g. Chapter X of [JP]); the estimate that comes closest to our result is Proposition 2.5 in $[\mathrm{FR}]$. We shall nevertheless go through the proof in the easier cases as well, since we need the intermediate steps to handle the more difficult ones. For the upper bounds we need to guess the curves that are "almost geodesic", i.e., give the value of the distance function up to additive constants, and integrate along such curves. Lower bounds are harder to obtain - we have to show that the curves guessed before are really the ones essentially minimizing the length in our metric.

Now let $x, y \in \Omega$ be given. Denote by $p=\pi(x)$ and $q=\pi(y)$ the projections of these points to the boundary.

Case 1: $x, y \in K$.

In this case $0 \leq d_{F}(x, y) \leq C$ and $0 \leq g(x, y) \leq C$, so (4.1) is trivially true.

Case 2: $x \in N, y \in K$, or $x \in K, y \in N$.

We may assume $x \in N, y \in K$. In this case $h(x) \vee h(y)=h(y) \geq \sqrt{\epsilon_{0}}$. Hence

$$
g(x, y)=\log (1 / h(x)) \pm C .
$$

To get an upper bound for $d_{F}(x, y)$ let $x^{\prime}=p-\epsilon_{0} n(p)$. By Lemma 2.1, we have $x^{\prime} \in K, \pi\left(x^{\prime}\right)=p$ and $x \in\left(x^{\prime}, p\right)$. Consider the segment $\gamma(t)=x+t\left(x^{\prime}-x\right)$, $t \in[0,1]$. Then Lemma 4.1 shows that

$$
d_{F}\left(x, x^{\prime}\right)=\log (1 / h(x)) \pm C .
$$

Since $d_{F}(x, y) \leq d_{F}\left(x, x^{\prime}\right)+d_{F}\left(x^{\prime}, y\right)$, and as $x^{\prime}, y \in K$ we have $d_{F}\left(x^{\prime}, y\right) \leq C$, this gives

$$
d_{F}(x, y) \leq \log (1 / h(x))+C .
$$

Together with (4.2) we get the right half of (4.1).

To obtain a lower bound for $d_{F}(x, y)$ let $\gamma$ be an arbitrary piecewise $C^{1}$-smooth curve in $\Omega$ joining $x$ and $y$. As we travel along $\gamma$ starting at $x$, there is a first point $y^{\prime}$ on the curve with $y^{\prime} \in K$. Then $h\left(y^{\prime}\right)=\sqrt{\epsilon_{0}}$. Let $\beta$ be the subcurve of $\gamma$ with endpoints $x$ and $y^{\prime}$. Then $\beta \subseteq \bar{N}$ and Lemma 4.1 shows

$$
F \text {-length }(\gamma) \geq F \text {-length }(\beta) \geq \log (1 / h(x))-C \text {. }
$$


Taking the infimum over all possible curves $\gamma$ in this inequality, we see that

$$
d_{F}(x, y) \geq \log (1 / h(x))-C .
$$

By (4.2) the left half of (4.1) follows.

Case 3: $x, y \in N, h(x) \vee h(y) \geq d_{H}(p, q)$.

We may assume $h(y) \geq h(x)$. Then

$$
g(x, y)=\log \left(\frac{h(y)}{h(x)}\right) \pm C .
$$

As in the second part of the previous case Lemma 4.1 shows that

$$
d_{F}(x, y) \geq \log \left(\frac{h(y)}{h(x)}\right)-C .
$$

To get an upper bound for $d_{F}(x, y)$ let $x^{\prime}$ be the unique point in $\pi^{-1}(p) \cap N$ with the same height as $y$, i.e., $x^{\prime}=p-\delta(y) n(p)$. Applying Lemma 4.1 to the line segment $\left[x, x^{\prime}\right]$ it follows that

$$
d_{F}\left(x, x^{\prime}\right) \leq \log \left(\frac{h\left(x^{\prime}\right)}{h(x)}\right)+C=\log \left(\frac{h(y)}{h(x)}\right)+C .
$$

It remains to find an upper bound for $d_{F}\left(x^{\prime}, y\right)$. By definition of $d_{H}(p, q)$ there exists a piecewise $C^{1}$-smooth horizontal curve $\alpha:[0,1] \rightarrow \partial \Omega$ with $\alpha(0)=p$, $\alpha(1)=q$, and $L_{\rho}$-length $(\alpha) \leq 2 d_{H}(p, q)$. Let us "lift" $\alpha$ to level $\delta(y)$ by defining $\gamma:[0,1] \rightarrow \Omega$ to be

$$
\gamma(t)=\alpha(t)-\delta(y) n(\alpha(t)), t \in[0,1]
$$

Note that $\gamma$ is piecewise $C^{1}$-smooth, $\gamma(0)=x^{\prime}, \gamma(1)=y$, and $\delta(\gamma(t))=\delta(y)$ for $t \in[0,1]$. Using (1.1) and (1.3) we can estimate for a.e. $t \in[0,1]$

$$
F(\gamma(t) ; \dot{\gamma}(t))^{2} \leq C\left(\frac{\left|\dot{\gamma}_{H}(t)\right|^{2}}{\delta(y)}+\frac{\left|\dot{\gamma}_{N}(t)\right|^{2}}{4 \delta(y)^{2}}\right)
$$

Next we apply the result of Lemma 2.2. Namely, we use (1.1), (2.3), (2.10), and the fact that $\alpha$ is a horizontal curve to obtain for a.e. $t$

$$
F(\gamma(t) ; \dot{\gamma}(t))^{2} \leq C \frac{|\dot{\alpha}(t)|^{2}}{\delta(y)}=C \frac{\left|\dot{\alpha}_{H}(t)\right|^{2}}{\delta(y)} \leq C \frac{L_{\rho}\left(\alpha(t) ; \dot{\alpha}_{H}(t)\right)}{\delta(y)}
$$

By integration this gives

$$
d_{F}\left(x^{\prime}, y\right) \leq F \text {-length }(\gamma) \leq \frac{C}{h(y)} L_{\rho} \text {-length }(\alpha) \leq C \frac{d_{H}(p, q)}{h(y)}
$$


Recall that $d_{H}(p, q) \leq h(y)$, and so $d_{F}\left(x^{\prime}, y\right) \leq C$ follows. Using (4.4) we obtain

$$
d_{F}(x, y) \leq d_{F}\left(x, x^{\prime}\right)+d_{F}\left(x^{\prime}, y\right) \leq \log \left(\frac{h(y)}{h(x)}\right)+C
$$

concluding the proof in this case.

Case 4: $x, y \in N, h(x) \vee h(y)<d_{H}(p, q)$.

We have

$$
g(x, y)=2 \log \left(\frac{d_{H}(p, q)}{\sqrt{h(x) h(y)}}\right) \pm C .
$$

Introduce $h_{0}:=\sqrt{\epsilon_{0}} \wedge d_{H}(p, q)$ where $a \wedge b=\min \{a, b\}$, and let $x^{\prime}=p-h_{0}^{2} n(p)$, $y^{\prime}=q-h_{0}^{2} n(q)$. Note that $C d_{H}(p, q) \leq h_{0} \leq d_{H}(p, q)$. As in Case 2 and Case 3 it follows that

$$
\begin{aligned}
& d_{F}\left(x, x^{\prime}\right) \leq \log \left(\frac{h\left(x^{\prime}\right)}{h(x)}\right)+C=\log \left(\frac{h_{0}}{h(x)}\right)+C, \\
& d_{F}\left(y, y^{\prime}\right) \leq \log \left(\frac{h\left(y^{\prime}\right)}{h(y)}\right)+C=\log \left(\frac{h_{0}}{h(y)}\right)+C .
\end{aligned}
$$

Furthermore, as in Case 3 it can be seen that $d_{F}\left(x^{\prime}, y^{\prime}\right) \leq C$. Therefore,

$$
d_{F}(x, y) \leq 2 \log \left(\frac{h_{0}}{\sqrt{h(x) h(y)}}\right)+C .
$$

From $h_{0} \leq d_{H}(p, q)$ and (4.5) the second inequality in (4.1) follows.

Before we go any further let us mention that we have in fact guessed an "almost" geodesic connecting $x$ and $y$. This is constructed as the union $\gamma_{x, x^{\prime}} \cup \gamma_{x^{\prime}, y^{\prime}} \cup \gamma_{y^{\prime}, y}$. Here $\gamma_{x, x^{\prime}}$ is a "vertical" segment going up from $x$ to $x^{\prime}$, i.e., to height level $h_{0}$ which is determined by $d_{H}(p, q)$. Similarly, the curve $\gamma_{y^{\prime}, y}$ is a vertical segment joining $y$ and $y^{\prime}$. The curve $\gamma_{x^{\prime}, y^{\prime}}$ is a "lift" to level $h_{0}$ of a horizontal curve which is almost a geodesic. The more difficult task is to get a lower bound for $d_{F}(x, y)$ in the present case. This amounts to showing that our guess above is indeed correct. We have to consider an arbitrary piecewise $C^{1}$-smooth curve $\gamma:[0,1] \rightarrow \Omega$ with $\gamma(0)=x$, $\gamma(1)=y$ and prove the right lower bound for its $F$-length. Given such a curve $\gamma$ define $H:=\max _{z \in \gamma} h(z)$. There exists $t_{0} \in[0,1]$ such that $H=h\left(\gamma\left(t_{0}\right)\right)$. Consider the two subcurves $\gamma_{1}=\gamma \mid\left[0, t_{0}\right]$ and $\gamma_{2}=\gamma \mid\left[t_{0}, 1\right]$. There are two possibilities.

If $H \geq h_{0}$ we obtain from Lemma 4.1 as in Case 2 that

$$
F \text {-length }\left(\gamma_{1}\right) \geq \log \left(\frac{h_{0}}{h(x)}\right)-C,
$$

and

$$
F \text {-length }\left(\gamma_{2}\right) \geq \log \left(\frac{h_{0}}{h(y)}\right)-C \text {. }
$$


Consequently, since $h_{0} \geq C d_{H}(p, q)$,

$$
F \text {-length }(\gamma) \geq 2 \log \left(\frac{d_{H}(p, q)}{\sqrt{h(x) h(y)}}\right)-C \text {. }
$$

The other possibility is when $H<h_{0}$. We then have $\gamma_{1} \cup \gamma_{2}=\gamma \subseteq N$. Let $\alpha=\pi \circ \gamma$ be the projection of $\gamma$ to the boundary. Since $h(x) \leq H$, there exists $k \in \mathbf{N}, k \geq 1$, such that $2^{-k} H<h(x) \leq 2^{-(k-1)} H$. Consider the curve $\gamma_{1}$ and define $0=s_{0} \leq s_{1}<\cdots<s_{k} \leq t_{0}$ as follows. Let $s_{0}=0$ and $s_{j}=\min \{s \in$ $\left.\left[0, t_{0}\right]: h(\gamma(s))=2^{-(k-j)} H\right\}$ for $j=1, \ldots, k$. Put $x_{j}=\gamma\left(s_{j}\right)$ and $p_{j}=\pi\left(x_{j}\right)$ for $j=0, \ldots, k$. Note that $1 \leq h\left(x_{j}\right) / h\left(x_{j-1}\right) \leq 2$ for $j=1, \ldots, k$.

We shall consider two alternatives. In the first case we assume that there exists an index $l \in\{1, \ldots, k\}$ such that

$$
d_{H}\left(p_{l-1}, p_{l}\right)>\frac{1}{8} 2^{-(k-l)} d_{H}(p, q) .
$$

Define the constant $\kappa>0$ such that

$$
\kappa^{-1}=\frac{1}{8}\left[2^{-(k-l)} d_{H}(p, q)\right]<d_{H}\left(p_{l-1}, p_{l}\right) .
$$

Then for $t \in\left[s_{l-1}, s_{l}\right]$ we have

$$
h(\gamma(t)) \leq 2^{-(k-l)} H \leq 2^{-(k-l)} d_{H}(p, q) \leq 8 / \kappa .
$$

Since $1-C_{1} \delta(x)^{s} \geq 1 / 2$ for $x \in N$, we obtain from (1.3) and Lemma 2.3

$$
\begin{aligned}
F \text {-length }\left(\gamma \mid\left[s_{l-1}, s_{l}\right]\right) & \geq C \int_{s_{l-1}}^{s_{l}} \frac{1}{h(\gamma(t))}\left[L_{\rho}\left(\alpha(t) ; \dot{\alpha}_{H}(t)\right)+\frac{\left|\dot{\alpha}_{N}(t)\right|^{2}}{h(\gamma(t))^{2}}\right]^{1 / 2} d t \\
& \geq C \frac{2^{k-l}}{H} \int_{s_{l-1}}^{s_{l}}\left(L_{\rho}\left(\alpha(t) ; \dot{\alpha}_{H}(t)\right)+\kappa^{2}\left|\dot{\alpha}_{N}(t)\right|^{2}\right)^{1 / 2} d t \\
& \geq C \frac{2^{k-l}}{H} d_{\kappa}\left(p_{l-1}, p_{l}\right) .
\end{aligned}
$$

Here $d_{\kappa}$ is the metric from the Approximation Lemma. An application of this lemma gives

$$
F \text {-length }\left(\gamma \mid\left[s_{l-1}, s_{l}\right]\right) \geq C \frac{d_{H}(p, q)}{H} .
$$

Let $t_{1}:=s_{k} \leq t_{0}$. As a consequence of Lemma 4.1 and (4.7) we have

$$
\begin{aligned}
F \text {-length }\left(\gamma \mid\left[0, t_{1}\right]\right)= & F \text {-length }\left(\gamma \mid\left[0, s_{l-1}\right]\right)+F \text {-length }\left(\gamma \mid\left[s_{l-1}, s_{l}\right]\right) \\
& +F \text {-length }\left(\gamma \mid\left[s_{l}, s_{k}\right]\right) \\
\geq & \log \left(\frac{h\left(x_{l-1}\right)}{h\left(x_{0}\right)}\right)+C \frac{d_{H}(p, q)}{H}+\log \left(\frac{h\left(x_{k}\right)}{h\left(x_{l}\right)}\right)-C \\
= & \log \left(\frac{h\left(x_{k}\right)}{h\left(x_{0}\right)}\right)+C \frac{d_{H}(p, q)}{H}-C \\
= & \log \left(\frac{H}{h(x)}\right)+C \frac{d_{H}(p, q)}{H}-C .
\end{aligned}
$$


The second alternative is that

$$
d_{H}\left(p_{j-1}, p_{j}\right) \leq \frac{1}{8} 2^{-(k-j)} d_{H}(p, q)
$$

for all $j=1, \ldots, k$. This implies

$$
d_{H}\left(p, \pi\left(\gamma\left(t_{1}\right)\right)\right) \leq \sum_{j=1}^{k} d_{H}\left(p_{j-1}, p_{j}\right) \leq \frac{1}{4} d_{H}(p, q) .
$$

On the other hand, by Lemma 4.1 again we get as before that

$$
F \text {-length }\left(\gamma \mid\left[0, t_{1}\right]\right) \geq \log \left(\frac{H}{h(x)}\right)-C \text {. }
$$

Summarizing this discussion we obtain the following two possibilities

$$
F \text {-length }\left(\gamma \mid\left[0, t_{1}\right]\right) \geq \log \left(\frac{H}{h(x)}\right)+C \frac{d_{H}(p, q)}{H}-C,
$$

or

$$
F \text {-length }\left(\gamma \mid\left[0, t_{1}\right]\right) \geq \log \left(\frac{H}{h(x)}\right)-C, \text { and } d_{H}\left(p, \pi\left(\gamma\left(t_{1}\right)\right)\right) \leq \frac{1}{4} d_{H}(p, q),
$$

where $t_{1} \in\left[0, t_{0}\right]$.

Applying similar considerations to the curve $\gamma_{2}$ instead of $\gamma_{1}$ we find $t_{2} \in\left[t_{0}, 1\right]$ such that one of the following alternatives holds

$$
F \text {-length }\left(\gamma \mid\left[t_{2}, 1\right]\right) \geq \log \left(\frac{H}{h(y)}\right)+C \frac{d_{H}(p, q)}{H}-C,
$$

or

$$
F \text {-length }\left(\gamma \mid\left[t_{2}, 1\right]\right) \geq \log \left(\frac{H}{h(y)}\right)-C, \text { and } d_{H}\left(q, \pi\left(\gamma\left(t_{2}\right)\right)\right) \leq \frac{1}{4} d_{H}(p, q) .
$$

Let us suppose that (A2) and (B2) hold simultaneously. Then

$d_{H}\left(\pi\left(\gamma\left(t_{1}\right)\right), \pi\left(\gamma\left(t_{2}\right)\right)\right) \geq d_{H}(p, q)-d_{H}\left(p, \pi\left(\gamma\left(t_{1}\right)\right)\right)-d_{H}\left(q, \pi\left(\gamma\left(t_{2}\right)\right)\right) \geq \frac{1}{2} d_{H}(p, q)$.

Again we can apply the Approximation Lemma (analogous to the case $l=k$ above) to conclude that

$$
F \text {-length }\left(\gamma \mid\left[t_{1}, t_{2}\right]\right) \geq C \frac{d_{H}(p, q)}{H}
$$


Consequently,

$$
\begin{aligned}
F \text {-length }(\gamma) & =F \text {-length }\left(\gamma \mid\left[0, t_{1}\right]\right)+F \text {-length }\left(\gamma \mid\left[t_{1}, t_{2}\right]\right)+F \text {-length }\left(\gamma \mid\left[t_{2}, 1\right]\right) \\
& \geq 2 \log \left(\frac{H}{\sqrt{h(x) h(y)}}\right)+C \frac{d_{H}(p, q)}{H}-C .
\end{aligned}
$$

This inequality (with an appropriate change of the constants $C$ if necessary) is also true if (A1) and (B1) or (A1) and (B2), or (A2) and (B1) hold simultaneously. In other words, (4.8) is true in any case. Elementary calculus shows that the right hand side expression of (4.8) considered as a function of $H$ has a minimum if $H$ is equal to $C d_{H}(p, q)$. This gives the lower bound

$$
F \text {-length }(\gamma) \geq 2 \log \left(\frac{d_{H}(p, q)}{\sqrt{h(x) h(y)}}\right)-C .
$$

If we take the infimum over all admissible curves $\gamma$, then (4.6), (4.9) and (4.5) show that $d_{F}(x, y) \geq g(x, y)-C$.

We have exhausted all the possibilities for $x$ and $y$, so (4.1) holds and the proof is complete.

As we pointed out in the introduction, Proposition 1.2 can be obtained by a slight modification of the argument in [M3]. Therefore, we will note repeat all the details, but just indicate the necessary adjustments.

Sketch of the proof of Proposition 1.2. We start from the following localization statement that relates the Kobayashi metric $K_{\Omega}$ on $\Omega$ with the Kobayashi metric $K_{\Omega \cap B(\pi(x), r)}$ on the intersection of $\Omega$ with a ball centered at a boundary point: There exist constants $\epsilon_{1}>0, r>0$, and $C>0$ such that for all $x \in \Omega$ with $\delta(x)<\epsilon_{1}$ and all $Z \in \mathbf{C}^{n}$ we have

$$
e^{-C \delta(x)} K_{\Omega \cap B(\pi(x), r)}(x, Z) \leq K_{\Omega}(x, Z) \leq K_{\Omega \cap B(\pi(x), r)}(x, Z) .
$$

This follows from [FR] as indicated on p. 333 of [M3]. By (4.10) it is enough to estimate the Kobayashi metric of the local domain $\Omega \cap B(\pi(x), r)$. To do this we apply a biholomorphism $\Psi: \Omega \cap B(\pi(x), r) \rightarrow \mathbf{C}^{n}$ with $\Psi(x)=0$ as given by formula (2.10) on p. 334 of [M3]. The next essential step in the argument of [M3] is to show that the image $\Psi(\Omega \cap B(\pi(x), r))$ is trapped between the two balls

$$
B\left(0, e^{-C \delta^{1 / 2}(x)}\right) \subseteq \Psi(\Omega \cap B(\pi(x), r)) \subseteq B\left(0, e^{C \delta^{1 / 2}(x)}\right)
$$

for some uniform constant $C=C(\Omega)$. The proof of (4.11) uses that the boundary is $C^{3}$-smooth and there seems to be no way to get this estimate with just the $C^{2}$-smooth boundary assumption. The appropriate modification is to show that 
the image $\Psi(\Omega \cap B(\pi(x), r))$ is squeezed between two complex ellipsoids. More precisely let us assume that $H_{\pi(x)} \partial \Omega=\{0\} \times \mathbf{C}^{n-1}$. Assuming the $C^{2}$-smoothness of $\partial \Omega$ one can show that

$$
E_{-} \subseteq \Psi(\Omega \cap B(\pi(x), r)) \subseteq E_{+},
$$

where $E_{ \pm}$are two complex ellipsoids given by

$$
E_{+}=\left\{y \in \mathbf{C}^{n}: e^{-C \delta^{1 / 2}(x)}\left|y_{1}\right|^{2}+e^{-\epsilon}\left(\left|y_{2}\right|^{2}+\ldots+\left|y_{n}\right|^{2}\right)<1\right\}
$$

and

$$
E_{-}=\left\{y \in \mathbf{C}^{n}: e^{C \delta^{1 / 2}(x)}\left|y_{1}\right|^{2}+e^{\epsilon}\left(\left|y_{2}\right|^{2}+\ldots+\left|y_{n}\right|^{2}\right)<1\right\} .
$$

The estimate (1.4) now follows from (4.12) by similar arguments as on pp. 335-336 of $[\mathrm{M} 3]$.

\section{Gromov hyperbolicity}

In this section we will discuss some background on Gromov hyperbolic spaces (cf. [BS], [GH], [G1]) and prove Theorem 1.4. Most of the results we need are discussed in [BS].

A metric space $X$ is called geodesic if any two points $x, y \in X$ can be joined by a geodesic segment. Any such geodesic segment, i.e., the image of a compact interval $I \subseteq \mathbf{R}$ under an isometry into $X$ will be denoted by $[x, y]$. The geodesic space is called $\delta$-hyperbolic if every geodesic triangle is $\delta$-thin, i.e., for any geodesic triangle $[x, y] \cup[y, z] \cup[x, z]$ in $X$ and any point $w \in[x, y]$ we have

$$
\operatorname{dist}(w,[y, z] \cup[x, z]) \leq \delta .
$$

A definition which works in general metric spaces is as follows. For a metric space $X$ denote the distance between two points $u$ and $v$ in $X$ by $|u-v|$, and define the Gromov product of two points $x, y \in X$ with respect to the basepoint $w \in X$ as $(x, y)_{w}=\frac{1}{2}\{|x-w|+|y-w|-|x-y|\}$. Then $X$ is called $\delta$-hyperbolic for $\delta \geq 0$ if

$$
(x, y)_{w} \geq(x, z)_{w} \wedge(z, y)_{w}-\delta \text { for all } \quad x, y, z, w \in X .
$$

For geodesic metric spaces $X$ the definitions are quantitatively equivalent, i.e., if $X$ is $\delta$-hyperbolic according to the first definition, then $X$ is $\delta^{\prime}$-hyperbolic according to the second definition with $\delta^{\prime}=\delta^{\prime}(\delta)$, and vice versa. We refer to Chapter 2 of $[\mathrm{GH}]$ for a detailed discussion of this equivalence.

The first definition is perhaps more frequently used in the literature, however we shall work with the second definition as it is better suited for our purposes. 
The concept of Gromov hyperbolicity can now be formulated. A metric space $X$ is called Gromov hyperbolic if it is $\delta$-hyperbolic for some $\delta \geq 0$.

A set $A$ in a metric space $X$ is called $k$-cobounded for $k \geq 0$ if every point $x \in X$ has distance at most $k$ from $A$. If $A$ is $k$-cobounded for some $k \geq 0$, then we say that is cobounded. Similarly, we will suppress mentioning the parameters of the notions defined below if the values of the parameters do not matter.

Definition 5.1. Let $f: X \rightarrow Y$ be a map (not necessarily continuous) between metric spaces $X$ and $Y$ and let $k \geq 0, \lambda \geq 1$ be constants. Suppose that $f(X)$ is $k$-cobounded in $Y$. If in addition for all $x, y \in X$

$$
|x-y|-k \leq|f(x)-f(y)| \leq|x-y|+k,
$$

then $f$ is a $k$-rough isometry; if

$$
\lambda|x-y|-k \leq|f(x)-f(y)| \leq \lambda|x-y|+k,
$$

then $f$ is a $(\lambda, k)$-rough similarity; if

$$
(1 / \lambda)|x-y|-k \leq|f(x)-f(y)| \leq \lambda|x-y|+k,
$$

then $f$ is a $(\lambda, k)$-rough quasiisometry.

Definition 5.2. Let $f: X \rightarrow Y$ be a bijection between metric spaces, and $\lambda \geq 1$, $\alpha>0$ be constants. If for all $x, y \in X$

$$
(1 / \lambda)|x-y| \leq|f(x)-f(y)| \leq \lambda|x-y|,
$$

then $f$ is $\lambda$-bilipschitz; if

$$
(1 / \lambda)|x-y|^{\alpha} \leq|f(x)-f(y)| \leq \lambda|x-y|^{\alpha},
$$

then $f$ is an $(\alpha, \lambda)$-snowflake map.

If for all distinct points $x, y, z \in X$

$$
\frac{|f(x)-f(z)|}{|f(x)-f(y)|} \leq \eta_{\alpha, \lambda}\left(\frac{|x-z|}{|x-y|}\right)
$$

then $f$ is an $(\alpha, \lambda)$-power quasisymmetry. Here we have used the notation

$$
\eta_{\alpha, \lambda}(t)=\left\{\begin{array}{cl}
\lambda t^{1 / \alpha} & \text { for } 0<t<1 \\
\lambda t^{\alpha} & \text { for } t \geq 1
\end{array}\right.
$$

For a Gromov hyperbolic space $X$ one can define a boundary set $\partial_{G} X$ as follows. Fix a basepoint $w \in X$. A sequence $\left(x_{i}\right)$ in $X$ is said to converge at infinity if 
$\lim _{i, j \rightarrow \infty}\left(x_{i}, x_{j}\right)_{w}=\infty$. Two sequences $\left(x_{i}\right)$ and $\left(y_{i}\right)$ converging at infinity are called equivalent if $\lim _{i \rightarrow \infty}\left(x_{i}, y_{i}\right)_{w}=\infty$. These notions do not depend on the choice of the basepoint. The boundary $\partial_{G} X$ is now defined as the set of equivalence classes of sequences converging at infinity. For $a, b \in \partial_{G} X$ and $w \in X$ we define

$$
(a, b)_{w}=\sup \liminf _{i \rightarrow \infty}\left(x_{i}, y_{i}\right)_{w} \in(0, \infty]
$$

where the supremum is taken over all sequences $\left(x_{i}\right)$ and $\left(y_{i}\right)$ representing the boundary points $a$ and $b$, respectively. The boundary $\partial_{G} X$ carries a canonical class of metrics. For any metric $d$ in this class there exists $\epsilon>0$ and $w \in X$ such that

$$
d(a, b) \asymp \exp \left(-\epsilon(a, b)_{w}\right) \quad \text { for } \quad a, b \in \partial_{G} X .
$$

Here we write $f \asymp g$ for two functions if there exists a constant $C \geq 1$ such that $(1 / C) f \leq g \leq C f$. Any two metrics $d_{1}$ and $d_{2}$ in the canonical class are snowflake equivalent, i.e., the identity map id: $\left(\partial_{G} X, d_{1}\right) \rightarrow\left(\partial_{G} X, d_{2}\right)$ is a snowflake map. One can define a topology on $X \cup \partial_{G} X$ that defines a compactification of the space $X$. This topology restricted to $\partial_{G} X$ agrees with the topology defined by the class of canonical metrics on the boundary.

The relevance of the maps defined in Definitions 5.1 and 5.2 in the context of Gromov hyperbolic spaces is due to the following proposition (cf. [BS], Sec. 6).

Proposition 5.3. Suppose $f: X \rightarrow Y$ is a rough quasiisometry between Gromov hyperbolic spaces $X$ and $Y$. Then $f$ induces a power quasisymmetry $\tilde{f}: \partial_{G} X \rightarrow$ $\partial_{G} Y$. If $f$ is a rough similarity, then $\tilde{f}$ is a snowflake map. If $f$ is a rough isometry and the boundaries $\partial_{G} X$ and $\partial_{G} Y$ are equipped with metrics satisfying an inequality as in (5.3) with the same number $\epsilon>0$, then $\tilde{f}$ is bilipschitz.

Note that for the validity of the first two statements it does not matter which metrics on $\partial_{X} G$ and $\partial_{G} Y$ we choose as long as they belong to the canonical class of metrics on the boundary. The induced map $\tilde{f}$ is defined by assigning to each sequence $\left(x_{i}\right)$ in $X$ converging at infinity the image sequence $\left(f\left(x_{i}\right)\right)$. It can be shown that $f$ is well-defined as a map from $\partial_{G} X$ to $\partial_{G} Y$.

The content of Proposition 5.3 can be summarized saying that a map $f: X \rightarrow$ $Y$ between Gromov hyperbolic spaces induces a map $\tilde{f}: \partial_{G} X \rightarrow \partial_{G} Y$ on their boundaries so that we have the following correspondence of maps:

$$
\begin{array}{ccc}
\text { rough isometry } & \longrightarrow & \text { bilipschitz map } \\
\text { rough similarity } & \longrightarrow & \text { snowflake map } \\
\text { rough quasiisometry } & \longrightarrow & \text { power quasisymmetry }
\end{array}
$$

The construction of the boundary can be reversed in the following way. Suppose $(Z, d)$ is a bounded metric space containing more that one point, and let $D(Z)>0$ 
be the diameter of $Z$. Define $\operatorname{Con}(Z)=Z \times(0, D(Z)]$, and for $(x, s),(y, t) \in$ $\operatorname{Con}(Z)$ let

$$
r((x, s),(y, t))=2 \log \left[\frac{d(x, y)+s \vee t}{\sqrt{s t}}\right] .
$$

Then it can be shown (cf. [BS], Sec. 7) that $r$ is a metric on $\operatorname{Con}(Z)$ such that $(\operatorname{Con}(Z), r)$ is Gromov hyperbolic. If $Z$ is complete, then the boundary $\partial_{G} \operatorname{Con}(Z)$ can be identified with $Z$ such that the metric $d$ is in the canonical class of snowflake equivalent metrics on $\partial_{G} \operatorname{Con}(Z)$. The expression in (5.4) has motivated our formula (1.2) of $g$.

We can now turn to the

Proof of Theorem 1.4. The proof that $\left(\Omega, d_{K}\right)$ is Gromov hyperbolic is very similar to the proof of Theorem 7.2 in [BS]. For the convenience of the reader we repeat the details.

Suppose we are given numbers $r_{i j} \geq 0$ such that $r_{i j}=r_{j i}$ and $r_{i j} \leq r_{i k}+r_{k j}$ for $i, j, k \in\{1,2,3,4\}$. Then $r_{12} r_{34} \leq 4\left(\left(r_{13} r_{24}\right) \vee\left(r_{14} r_{23}\right)\right)$. To see this, we may assume that $r_{13}$ is the smallest of the quantities $r_{i j}$ appearing on the right hand side of this inequality. Then $r_{12} \leq r_{13}+r_{32} \leq 2 r_{23}$ and $r_{34} \leq r_{31}+r_{14} \leq 2 r_{14}$. The inequality follows.

Now let $x_{i}, i \in\{1,2,3,4\}$, be four arbitrary points in $\Omega$, and denote by $p_{i}=$ $\pi\left(x_{i}\right)$ their projections to the boundary and by $h_{i}=\delta\left(x_{i}\right)^{1 / 2}$ their heights. Set $d_{i j}=d_{H}\left(p_{i}, p_{j}\right)$ and $r_{i j}=d_{i j}+h_{i} \vee h_{j}$. Then

$$
\begin{aligned}
& \left(d_{1,2}+h_{1} \vee h_{2}\right)\left(d_{3,4}+h_{3} \vee h_{4}\right) \\
& \quad \leq 4\left(\left(d_{1,3}+h_{1} \vee h_{3}\right)\left(d_{2,4}+h_{2} \vee h_{4}\right)\right) \vee\left(\left(d_{1,4}+h_{1} \vee h_{4}\right)\left(d_{2,3}+h_{2} \vee h_{3}\right)\right) .
\end{aligned}
$$

By Corollary 1.3 this translates to

$d_{K}\left(x_{1}, x_{2}\right)+d_{K}\left(x_{3}, x_{4}\right) \leq\left(d_{K}\left(x_{1}, x_{3}\right)+d_{K}\left(x_{2}, x_{4}\right)\right) \vee\left(d_{K}\left(x_{1}, x_{4}\right)+d_{K}\left(x_{2}, x_{3}\right)\right)+C$,

where $C$ is a constant independent of the points. This inequality is equivalent to the Gromov hyperbolicity of the space $\left(\Omega, d_{K}\right)$.

It follows from the definition and Corollary 1.3 that a sequence $\left(x_{i}\right)$ in $\left(\Omega, d_{K}\right)$ converges at infinity if and only if the sequence $\left(\pi\left(x_{i}\right)\right)$ converges and $h\left(x_{i}\right) \rightarrow$ 0 as $i \rightarrow \infty$. This happens if and only if $\left(x_{i}\right)$ converges with respect to the Euclidean metric to a point in $\partial \Omega$. Moreover, two sequences converging at infinity are equivalent if and only if their limit points on $\partial \Omega$ are the same. Each point in $\partial \Omega$ arises as a limit point of a sequence converging at infinity.

Assigning to each equivalence class of sequences in $\Omega$ converging at infinity the unique limit point of each sequence in the class, we can identify the Gromov boundary $\partial \Omega$ with the Euclidean boundary as sets.

Some straightforward calculation based on the definition of the Gromov product for boundary points and Corollary 1.3 shows that for any choice of a basepoint $w \in \Omega$ we have

$$
d_{H}(a, b) \asymp \exp \left(-(a, b)_{w}\right) \quad \text { for } \quad a, b \in \partial \Omega .
$$


This shows that the Carnot-Carathéodory metric $d_{H}$ is in the canonical class of snowflake equivalent metrics on $\partial_{G} \Omega=\partial \Omega$.

We give an example for a class of pseudoconvex domains that are not Gromov hyperbolic with the Kobayashi metric. Our examples are of product-type $\Omega=$ $\Omega_{1} \times \Omega_{2} \subseteq \mathbf{C}^{n_{1}} \times \mathbf{C}^{n_{2}}$.

Proposition 5.6. Let $\Omega_{1} \subseteq \mathbf{C}^{n_{1}}, \Omega_{2} \subseteq \mathbf{C}^{n_{2}}, n_{1}, n_{2} \geq 2$, be bounded strictly pseudoconvex domains with $C^{2}$-smooth boundary. Then the product domain $\Omega:=$ $\Omega_{1} \times \Omega_{2} \subseteq \mathbf{C}^{n_{1}+n_{2}}$ equipped with the Kobayashi distance is not Gromov hyperbolic.

Proof. Denote by $d, d_{1}$ and $d_{2}$ the Kobayashi distance on $\Omega, \Omega_{1}$ and $\Omega_{2}$, respectively. The proof is based on the following product formula (cf. [JP], p. 107)

$$
d\left(\left(x_{1}, y_{1}\right),\left(x_{2}, y_{2}\right)\right)=\max \left\{d_{1}\left(x_{1}, x_{2}\right), d_{2}\left(y_{1}, y_{2}\right)\right\} .
$$

Let us assume that $(\Omega, d)$ is a Gromov hyperbolic space. In particular (5.1) holds for some $\delta>0$. Let us introduce $k:=3+2 \delta$ and choose two points $x_{1}, x_{2} \in \Omega_{1}$ such that $d_{1}\left(x_{1}, x_{2}\right)=2 k$. Select a third point $x_{3} \in \Omega_{1}$ such that

$$
k \leq d_{1}\left(x_{1}, x_{3}\right) \leq d_{1}\left(x_{3}, x_{2}\right) \leq k+1 .
$$

Fix $y_{1} \in \Omega_{2}$, and consider the three points $x=\left(x_{1}, y_{1}\right), y=\left(x_{2}, y_{1}\right), w=$ $\left(x_{3}, y_{1}\right) \in \Omega$. Using (5.6) and (5.7) we can see that the Gromov product $(x, y)_{w}$ satisfies $(x, y)_{w} \leq 1$. Choose a fourth point $z \in \Omega$ of the form $z=\left(x_{3}, y_{2}\right)$, where $y_{2} \in \Omega_{2}$ is such that $d_{2}\left(y_{1}, y_{2}\right)=2 k$. Using (5.6) again we see that

$$
d(z, w)=d(z, x)=d(z, y)=2 k .
$$

Hence for the corresponding Gromov products we obtain that

$$
(z, x)_{w}=\frac{1}{2} d(x, w) \geq \frac{k}{2} \text { and }(z, y)_{w}=\frac{1}{2} d(y, w) \geq \frac{k}{2} .
$$

By the definition of $k$ and since $(x, y)_{w} \leq 1$, relations (5.8) are in contradiction to (5.1) proving the proposition.

\section{Applications and final remarks}

As we pointed out in the introduction, the theory of Gromov hyperbolic spaces gives a general framework for the theory of boundary extensions for biholomorphisms and proper holomorphic maps. We have seen in Proposition 5.3 that isometries between Gromov hyperbolic spaces, and more general, rough quasiisometries induce homeomorphisms on the boundaries of these spaces. 
According to Theorem 1.4 we can apply these general facts to the situation when our metric spaces are strictly pseodoconvex domains equipped with the Kobayashi distance. Hence we get the following result.

Corollary 6.1. Let $\Omega_{1}, \Omega_{2} \subseteq \mathbf{C}^{n}, n \geq 2$, be bounded strictly pseudoconvex domains with $C^{2}$-smooth boundary. Let $f: \Omega_{1} \rightarrow \Omega_{2}$ be a continuous mapping that is a rough quasiisometry with respect to the Kobayashi distances on the domains. Then $f$ has a continuous extension $\bar{f}: \bar{\Omega}_{1} \rightarrow \bar{\Omega}_{2}$ such that the induced boundary map $\tilde{f}:=\left.\bar{f}\right|_{\partial \Omega_{1}}: \partial \Omega_{1} \rightarrow \partial \Omega_{2}$ is a power quasisymmetry with respect to the CarnotCarathéodory metrics. Moreover, if $f$ is a rough similarity, then $\tilde{f}$ is a snowflake map; if $f$ is a rough isometry, then $\tilde{f}$ is bilipschitz.

In order to get the last statement one has to observe that by (5.5) a formula of type (5.3) is valid for the Carnot-Carathéodory metrics on the boundary of the regions with the same $\epsilon$, namely $\epsilon=1$.

Since biholomorphisms are isometries of the Kobayashi metric, they induce maps on the boundary that are bilipschitz maps in the Carnot-Carathéodory metrics. Corollary 6.1 is therefore a version of the celebrated boundary extension phenomenon of biholomorphisms [Fef] in the sense of coarse geometry. For the case of proper mappings we have the following statement.

Corollary 6.2. Let $\Omega_{1}, \Omega_{2} \subseteq \mathbf{C}^{n}, n \geq 2$, be bounded strictly pseudoconvex domains with $C^{2}$-smooth boundary, and let $f: \Omega_{1} \rightarrow \Omega_{2}$ be a proper holomorphic map. Then $f$ extends continuously to a map $\bar{f}: \bar{\Omega}_{1} \rightarrow \bar{\Omega}_{2}$ with $f\left(\partial \Omega_{1}\right) \subseteq \partial \Omega_{2}$. The induced map $\tilde{f}:=\left.\bar{f}\right|_{\partial \Omega_{1}}: \partial \Omega_{1} \rightarrow \partial \Omega_{2}$ is a Lipschitz map if we equip the boundaries of the domains with the Carnot-Carathéodory metrics.

It is well-known that the extension $\bar{f}: \bar{\Omega}_{1} \rightarrow \bar{\Omega}_{2}$ is Hölder continuous with exponent $1 / 2$. This follows easily from Corollary 6.2 and the relation (3.1) between the Carnot-Carathéodory and Euclidean metric on $\partial \Omega$. Various other regularity results can be found in literature (cf. $[\mathrm{Co}],[\mathrm{Kh}],[\mathrm{Le}],[\mathrm{Pi}],[\mathrm{PK}])$, however it seems not to have been observed before that $f: \partial \Omega_{1} \rightarrow \partial \Omega_{2}$ is Lipschitz if the boundaries carry the Carnot-Carathéodory metrics.

Proof. Let $f: \Omega_{1} \rightarrow \Omega_{2}$ be a proper holomorphic mapping. For $i \in\{1,2\}$ let $\delta_{i}(x)=\operatorname{dist}\left(x, \partial \Omega_{i}\right), x \in \mathbf{C}^{n}$, let $K_{i}$ be the Kobayashi metric on $\Omega_{i}$ and $d_{i}$ the distance function associated with $K_{i}$, and let $d_{H}^{i}$ be the Carnot-Carathéodory metric on $\partial \Omega_{i}$. Then we have for all $x \in \Omega_{1}$ and $Z \in \mathbf{C}^{n}$

$$
K_{2}(f(x) ; D f(x) Z) \leq K_{1}(x ; Z) .
$$

Here $D f(x)$ is the tangential map of $f$ at $x$. This implies that for all $x, y \in \Omega_{1}$ we have

$$
d_{2}(f(x), f(y)) \leq d_{1}(x, y) .
$$


Since $f$ is proper there exists a constant $C_{1} \geq 1$ such that for all $x \in \Omega_{1}$ we have

$$
\left(1 / C_{1}\right) \delta_{1}(x) \leq \delta_{2}(f(x)) \leq C_{1} \delta_{1}(x) .
$$

From Corollary 1.3, and inequalities (6.1) and (6.2) we conclude that there exists a constant $C_{2}>0$ such that for all $x, y \in \Omega_{1}$

$$
d_{H}^{2}(\pi(f(x)), \pi(f(y))) \leq C_{2}\left(d_{H}^{1}(\pi(x), \pi(y))+\delta_{1}(x)^{1 / 2} \vee \delta_{1}(y)^{1 / 2}\right) .
$$

From (6.2) and (6.3) it follows that whenever a sequence in $\Omega_{1}$ converges to some point in $\partial \Omega_{1}$, then the image sequence under $f$ converges to a point $\partial \Omega_{2}$. Moreover, the image sequences of two sequences in $\Omega_{1}$ converging to the same boundary point in $\partial \Omega_{1}$ converge to the same boundary point in $\partial \Omega_{2}$. It follows that $f$ has a unique extension (also called $f$ ) to $\bar{\Omega}_{1}$ which is continuous which respect to the Euclidean metric. Moreover, $f\left(\partial \Omega_{1}\right) \subseteq \partial \Omega_{2}$ and from (6.3) we get for $a, b \in \partial \Omega_{1}$

$$
d_{H}^{2}(f(a), f(b)) \leq C_{2} d_{H}^{1}(a, b) .
$$

This shows that the boundary map is Lipschitz if the boundaries of the domains are equipped with the Carnot-Carathéodory metrics.

Let us mention that Corollary 6.2 would also follow from the stronger results as announced in $[\mathrm{Kh}]$. Nevertheless, our proof is rather elementary and illustrates well the efficiency of Corollary 1.3. Even though we do not get the best regularity possible in the immediate applications, our approach has the advantage that it works for much more general mappings (cf. Corollary 6.1).

Remarks: 1. Results as in Corollary 1.3 and Theorem 1.4 hold for the (inner-) Carathéodory distance and the Bergman distance as well, at least if we have some higher regularity of the boundary (this was announced in [BB]). In order to apply Theorem 1.1 we need analogs of Proposition 1.2 for the Carathéodory metric and the Bergman metric. If the boundary is sufficiently smooth, estimates like this occur explicitly in the literature (cf. [Ca], [Di1], [Di2], [Fu], [M1]). In the case of a $C^{2}$-smooth boundary the estimates corresponding to (1.4) are likely to be true, but it is not so straightforward to adapt arguments given in the literature. We hope to come back to this issue in a later paper. In [BB] we contended ourselves with a result slightly weaker than Corollary 1.3.

2. We would like to point out briefly how earlier estimates for the Kobayashi distance (cf. $[\mathrm{Ab}],[\mathrm{FR}],[\mathrm{JP}],[\mathrm{K}])$ follow as special cases of our Corollary 1.3.

When $x$ belongs to some fixed compact set $K \subseteq \Omega$, then

$$
d_{K}(x, y)=\frac{1}{2} \log \frac{1}{\delta(y)} \pm C(K) .
$$

In the case when $x, y \in \Omega$ are sufficiently close to two distinct points on $\partial \Omega$, then our formula (1.2) yields

$$
d_{K}(x, y)=\frac{1}{2} \log \frac{1}{\delta(x)}+\frac{1}{2} \log \frac{1}{\delta(y)} \pm C,
$$


which is another well-known result.

Let us also recall from $[\mathrm{FR}]$ an upper estimate for the Kobayashi distance of two point sufficiently close to a given boundary point

$$
d_{K}(x, y) \leq \frac{1}{2} \log \left(1+\frac{|x-y|}{\delta(x)}\right)+\frac{1}{2} \log \left(1+\frac{|x-y|}{\delta(y)}\right)+C,
$$

which also follows from (1.2) taking into account the upper bound for the CarnotCarathéodory metric in (3.1).

In fact (6.4) is the result that comes closest to ours but it is just an upper estimate. Our point is that the use of the Carnot-Carathéodory metric leads to precise two-sided estimates.

3. Naturally, one could wonder about possible extensions of our results. We think that the failure of Gromov hyperbolicity in Proposition 5.4 is due to the loss of strict pseudoconvexity rather than the loss of smoothness of the boundary. It seems likely that a smoothing procedure of the boundary of certain product domains should lead to pseudoconvex domains with smooth boundary that are not Gromov hyperbolic. In the positive direction, we think that to some extent we can give up strict pseudoconvexity as we conjecture that some of our results carry over to the class of domains of finite type, although possibly with considerable technical complications. In particular, it is likely that one has to use the full power of $[\mathrm{NSW}]$ to study the horizontal metric in this case (cf. Section 3). The recent results of $[\mathrm{BSY}],[\mathrm{KY}],[\mathrm{Ca}],[\mathrm{DO}],[\mathrm{DH}]$ encourage the investigation in this direction. It should also be possible to relax the smoothness condition. This is indicated by the fact that there is a large class of domains in $\mathbf{R}^{n}$ with non-smooth boundary that are Gromov hyperbolic with the quasihyperbolic metric (cf. [BHK]).

\section{Acknowledgement}

We would like to thank J.E. Fornæss, L. Lempert, S. Krantz, A. Korányi and M. Reimann for advice and discussions related to this paper. Most of this research was completed while the second author was visiting the University in Bern. He would like to thank the local Department of Mathematics for its hospitality.

\section{References}

[Ab] M. Abate, Boundary behavior of invariant distances and complex geodesics, Atti Accad. Naz. Lincei Rend. Cl. Sci. Fis. Mat. Natur. 80(8) (1986), 100-106.

[A1] G. Aladro, Localization of the Kobayashi distance, J. Math. Anal. Appl. 181 (1994), 200-204.

[BB] Z. M. Balogh, M. Bonk, Pseudoconvexity and Gromov hyperbolicity, C. R. Acad. Sci. Paris Sér. I Math. 328 (1999), 597-602.

[Be] A. Bellaïche, The tangent space in sub-Riemannian geometry. In: Sub-Riemannian Geometry (Eds. A. Bellaïche, J.-J. Risler), Progress in Math. 144, Birkhäuser, Basel 1996, pp. 1-78. 
[BHK] M. Bonk, J. Heinonen, P. Koskela, Uniformizing Gromov hyperbolic spaces, preprint.

[BS] M. Bonk, O. Schramm, Embeddings of Gromov hyperbolic spaces, Geom. Func. Anal. to appear.

[BSY] H. Boas, E. Straube, J. Yu, Boundary limits of the Bergman kernel and metric, Mich. Math. J. 42 (1995), 449-461.

[Ca] D. Catlin, Estimates of invariant metrics on pseudoconvex domains of dimension two, Math. Z. 200 (1989), 429-466.

[Co] B. Coupet, Precise regularity up to the boundary of proper holomorphic mappings, Ann. Scuola Norm. Sup. Pisa Cl. Sci. 20(4) (1993), 461-482.

[Di1] K. Diederich, Das Randverhalten der Bergmanschen Kernfunktion und Metrik in streng pseudo-konvexen Gebieten, Math. Ann. 187 (1970), 9-36.

[Di2] K. Diederich, Über die 1. und 2. Ableitungen der Bergmanschen Kernfunktion und ihr Randverhalten, Math. Ann. 203 (1973), 129-170.

[DH] K. Diederich, G. Herbort, Geometric and analytic boundary invariants on pseudoconvex domains. Comparison Results. J. Geom. Anal. 3 (1993), 237-267.

[DO] K. Diederich, T. Ohsawa, An estimate for the Bergman distance on pseudoconvex domains, Ann. Math. 141(2) (1995), 181-190.

[Fed] H. Federer, Curvature measures, Trans. Amer. Math. Soc. 93 (1959), 418-491.

[Fef] Ch. Fefferman, The Bergman kernel and biholomorphic mappings of pseudoconvex domains, Invent. Math. 26 (1974), 1-65.

[FR] F. Forstnerič, J.-P. Rosay, Localization of the Kobayashi metric and the boundary continuity of proper holomorphic mappings, Math. Ann. 279 (1987), 239-252.

[FS] G. B. Folland, E. M. Stein, Estimates for the $\bar{\partial}_{b}$ complex and analysis on the Heisenberg group, Comm. Pure Appl. Math. 27 (1974), 429-523.

[Fu] S. Fu, Asymptotic expansions of invariant metrics of strictly pseudoconvex domains, Canad. Math. Bull. 38 (1995), 196-206,

[GH] E. Ghys, P. de la Harpe (Eds.), Sur les groupes hyperboliques d'après Mikhael Gromov Progess in Math. 38 Birkhäuser, Boston 1990.

[Gr] I. Graham, Boundary behavior of the Carathéodory and Kobayashi metrics on strongly pseudoconvex domains in $\mathbf{C}^{n}$ with smooth boundary Trans. Amer. Math. Soc. 207 (1975), 219-240.

[G1] M. Gromov, Hyperbolic groups. In: Essays in group theory (Ed. S.M. Gersten), Math. Sci. Res. Inst. Publ. 8 Springer, New York 1987, pp. 75-263.

[G2] M. Gromov, Carnot-Carathéodory spaces seen from within. In: Sub-Riemannian Geometry (Eds. A. Bellaïche, J.-J. Risler), Progress in Math. 144 Birkhäuser, Basel 1996, pp. 79-323.

[JP] M. Jarnicki, P. Pflug, Invariant Distances and Metrics in Complex Analysis, Expo. in Math. 9, de Gruyter, Berlin 1993.

[Kh] Yu. V. Khurumov. Boundary smoothness of proper holomorphic mappings of strictly pseudoconvex domains (Russian) Mat. Zametki 48 (1990), 149-150.

[K] S. Kobayashi, Hyperbolic complex spaces, Grundlehren der math. Wissenschaften 318 Springer, Berlin 1998.

[Ko] A. Korányi, Geometric aspects of analysis on the Heisenberg group. In: Topics in Modern Harmonic Analysis, Proc. Semin. Torino and Milano 1982, Vol. I (1983), 209-259.

[K1] S. G. Krantz, Function Theory of Several Complex Variables, 2. ed., Cole Math. Series, Wadsworth \& Brooks, Pacific Grove, CA 1992.

[K2] S. G. Krantz, The boundary behavior of the Kobayashi metric Rocky Mount. J. Math. 22 (1992), 227-233.

[KP] S. G. Krantz, H. R. Parks, Distance to $C^{k}$ hypersurfaces J. Diff. Equ. 40 (1981), $116-120$.

[KY] S. G. Krantz, J. Yu, On the Bergman invariant and curvatures of the Bergman metric, 
Illinois J. Math. 40 (1996), 226-244.

[Le] L. Lempert, A precise result on the boundary regularity of biholomorphic mappings, Math. Z. 193 (1986), 559-579. Erratum: Math. Z. 206, (1991), 501-504

[M1] D. Ma, Boundary behavior of invariant metrics and volume forms on strongly pseudoconvex domains, Duke J. Math. 63 (1991), 673-697.

[M2] D. Ma, On iterates of holomorphic maps, Math. Z. 207 (1991), 417-428.

[M3] D. Ma, Sharp estimates of the Kobayashi metric near strongly pseudoconvex points. In: Madison Symposium on Complex Analysis, Contemporary Math. 137 Amer. Math. Soc., Providence, RI 1992, pp. 329-339.

[NSW] A. Nagel, E. M. Stein, S. Wainger, Balls and metrics defined by vector fields. I. Basic properties, Acta Math. 155 (1985), 103-147.

[Pi] S. I. Pinchuk, The scaling method and holomorphic mappings. In: Several complex variables and complex geometry, Part 1 (Santa Cruz, CA, 1989), Proc. Sympos. Pure Math. 52 Part 1 Amer. Math. Soc. Providence, RI 1991, pp. 151-161.

[PK] S. I. Pinchuk, S. V. Khasanov, Asymptotically holomorphic functions and their applications (Russian) Mat. Sb. (N.S.) 134 (176) (1987), 546-555, 576.

Zoltán M. Balogh

Universität Bern

Mathematisches Institut

Sidlerstr. 5

CH-3012 Bern

Switzerland

e-mail: zoltan@math-stat.unibe.ch
Mario Bonk

Institut für Analysis

Tech. Univ. Braunschweig

D-38106 Braunschweig

Germany

e-mail: M.Bonk@tu-bs.de

Current address:

University of Michigan

Department of Mathematics

Ann Arbor, MI 48109

USA

(Received: January 11, 2000) 